\title{
Constraints on the diffuse flux of ultrahigh energy neutrinos from four years of Askaryan Radio Array data in two stations
}

P. Allison $\odot,{ }^{1}$ S. Archambault, ${ }^{2}$ J. J. Beatty $\odot,{ }^{1}$ M. Beheler-Amass, ${ }^{3}$ D. Z. Besson $\odot,{ }^{4,5}$ M. Beydler, ${ }^{3}$ C. C. Chen, ${ }^{6}$ C. H. Chen, ${ }^{6}$ P. Chen, ${ }^{6}$ B. A. Clark $\odot,{ }^{1,7, *}$ W. Clay, ${ }^{8}$ A. Connolly, ${ }^{1}$ L. Cremonesi $\odot,{ }^{9}$ J. Davies, ${ }^{9}$ S. de Kockere $\odot,{ }^{10}$ K. D. de Vries $\odot,{ }^{10}$ C. Deaconu, ${ }^{8}$ M. A. DuVernois $\odot,{ }^{3}$ E. Friedman, ${ }^{11}$ R. Gaior, ${ }^{2}$ J. Hanson, ${ }^{12}$ K. Hanson, ${ }^{3}$ K. D. Hoffman, ${ }^{11}$ B. Hokanson-Fasig, ${ }^{3}$ E. Hong, ${ }^{1}$ S. Y. Hsu, ${ }^{6}$ L. Hu, ${ }^{6}$ J. J. Huang, ${ }^{6}$ M.-H. Huang, ${ }^{6}$ K. Hughes, ${ }^{8}$ A. Ishihara, ${ }^{2}$ A. Karle, ${ }^{3}$ J. L. Kelley $\odot,{ }^{3}$ R. Khandelwal, ${ }^{3}$ K.-C. Kim, ${ }^{11}$ M.-C. Kim $\odot,{ }^{2}$ I. Kravchenko, ${ }^{13}$ K. Kurusu, ${ }^{2}$ H. Landsman, ${ }^{14}$ U. A. Latif, ${ }^{4}$ A. Laundrie, ${ }^{3}$ C.-J. Li, ${ }^{6}$ T.-C. Liu, ${ }^{6}$ M.-Y. Lu $\odot,{ }^{3, \dagger}$ B. Madison, ${ }^{4}$ K. Mase, ${ }^{2}$ T. Meures, ${ }^{3}$ J. Nam, ${ }^{2}$ R. J. Nichol, ${ }^{9}$ G. Nir, ${ }^{14}$ A. Novikov $\odot,{ }^{4,5}$ A. Nozdrina $\odot,{ }^{4}$ E. Oberla, ${ }^{8}$ A. O'Murchadha, ${ }^{3}$ J. Osborn, ${ }^{13}$ Y. Pan, ${ }^{15}$ C. Pfendner $\odot,{ }^{16}$ J. Roth, ${ }^{15}$ P. Sandstrom, ${ }^{3}$ D. Seckel, ${ }^{15}$ Y.-S. Shiao, ${ }^{6}$ A. Shultz, ${ }^{4}$ D. Smith, ${ }^{8}$ J. Torres $\odot,{ }^{1,+}$ J. Touart, ${ }^{11}$ N. van Eijndhoven $\odot,{ }^{10}$ G. S. Varner, ${ }^{17}$ A. G. Vieregg, ${ }^{8}$ M.-Z. Wang, ${ }^{6}$ S.-H. Wang $\odot,{ }^{6}$ S. A. Wissel $\odot,{ }^{18,19}$ S. Yoshida, ${ }^{2}$ and R. Young ${ }^{4}$

(ARA Collaboration)

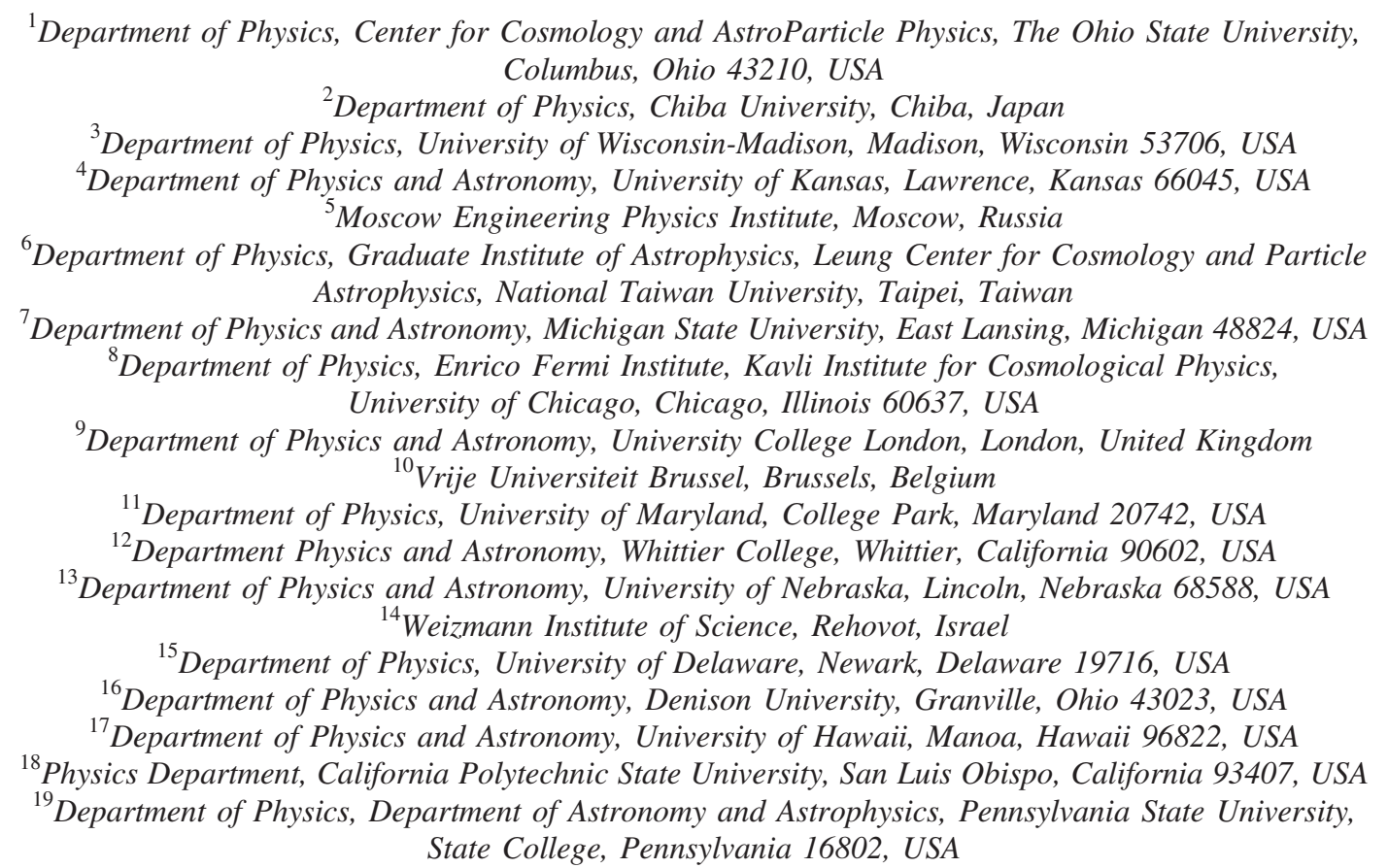

(Received 11 May 2020; accepted 15 July 2020; published 27 August 2020)

The Askaryan Radio Array (ARA) is an ultrahigh energy (UHE, $>10^{17} \mathrm{eV}$ ) neutrino detector designed to observe neutrinos by searching for the radio waves emitted by the relativistic products of neutrinonucleon interactions in Antarctic ice. In this paper, we present constraints on the diffuse flux of ultrahigh energy neutrinos between $10^{16}$ and $10^{21} \mathrm{eV}$ resulting from a search for neutrinos in two complementary analyses, both analyzing four years of data (2013-2016) from the two deep stations (A2, A3) operating at that time. We place a 90\% CL upper limit on the diffuse all flavor neutrino flux at $10^{18} \mathrm{eV}$ of $E F(E)=5.6 \times 10^{-16} \mathrm{~cm}^{-2} \mathrm{~s}^{-1} \mathrm{sr}^{-1}$. This analysis includes four times the exposure of the previous ARA result and represents approximately $1 / 5$ th the exposure expected from operating ARA until the end of 2022.

DOI: 10.1103/PhysRevD.102.043021

\footnotetext{
*baclark@msu.edu mlu27@wisc.edu

torresespinosa.1@osu.edu
} 


\section{INTRODUCTION}

Ultrahigh energy neutrinos (UHE, $>10^{17} \mathrm{eV}$ ) are a unique window on the distant, high-energy Universe. In addition to gravitational waves, they are the only Standard Model messengers capable of traveling cosmic distances undeflected and unattenuated. Cosmic rays have their trajectories bent by magnetic fields, and for sources more distant than $\sim 50 \mathrm{Mpc}$, above $\sim 10^{19.5} \mathrm{eV}$ cosmic rays are expected to be degraded in energy through interactions with the cosmic microwave background (CMB) via the GreisenZatsepin-Kuz'min effect [1,2]. Cosmic ray nuclei are additionally degraded in-flight to earth through their natural beta and inverse-beta decay processes, as well as photo disintegration, e.g., the giant dipole resonance [3]. Highenergy gamma rays $(\gtrsim 100 \mathrm{TeV})$ are similarly expected to pair-annihilate off the $\mathrm{CMB}$ and extragalactic background light [4].

Predictions for the sources of very high-energy neutrinos fall broadly into two classes. First, astrophysical neutrinos are expected from the site of cosmic ray acceleration, for example, gamma ray bursts and active galactic nuclei $[5,6]$. The IceCube experiment has confirmed the existence, and measured the spectrum, of TeV-PeV astrophysical neutrinos [7], and has identified a first potential source in the blazar TXS $0506+056[8,9]$. Second, cosmogenic neutrinos are expected from the destruction of cosmic rays through the aforementioned processes [10]. A more complete discussion of how the flux of cosmogenic neutrinos depends on the primary cosmic-ray composition, and the effects of various interaction and decay processes, can be found in the literature [11-15].

At energies above $10^{16} \mathrm{eV}$, low predicted fluxes $[16,17]$ combined with small expected cross sections $[18,19]$ lead to $\mathcal{O}\left(10^{-2}\right)$ neutrino interactions per cubic-kilometer of ice per year per energy decade. As such, the active volumes of the instruments required to detect this UHE flux must necessarily approach the scale of $100 \mathrm{~km}^{3}$ water equivalent. Several experiments are operating or under construction to reach this high-energy flux, including IceCube [20], Pierre Auger [21], NuMoon [22], ANITA [23], ARIANNA [24], GRAND [25], and ARA [26], which is the focus on this work.

The Askaryan Radio Array (ARA) is a UHE neutrino detector deployed at the South Pole seeking to observe these ultrahigh energy neutrinos. ARA searches for neutrinos by looking for the broadband (few hundred $\mathrm{MHz}$ to few $\mathrm{GHz}$ ) radio impulse, or "Askaryan emission" [27,28], that accompanies neutrino-nucleon interactions. This effect, caused by a 20\% negative charge asymmetry in electromagnetic showers in media, and acting as a coherently radiating current distribution, has been observed in the laboratory at accelerator facilities [29]. The radiation has a Cherenkov-like beam pattern, with a cone thickness of a few degrees. The leading edge of the electric field pulse points toward the shower axis. Experiments looking for Askaryan radiation are deployed in dielectric media such as ice, salt, and sand, which are expected to be sufficiently transparent to radio waves as to make the radio signal observable. In the case of ARA, the long (generally greater than $500 \mathrm{~m}$ [30]) attenuation length of radio waves in South Pole ice allows naturally occurring detector volumes to be instrumented sparsely and economically. A diagram of how a neutrino interaction might be observed in an ARA detector is given in Fig. 1.

In this paper, we report constraints on the diffuse flux of UHE neutrinos over the energy interval $10^{16}-10^{21} \mathrm{eV}$. This result is based on two complementary searches for

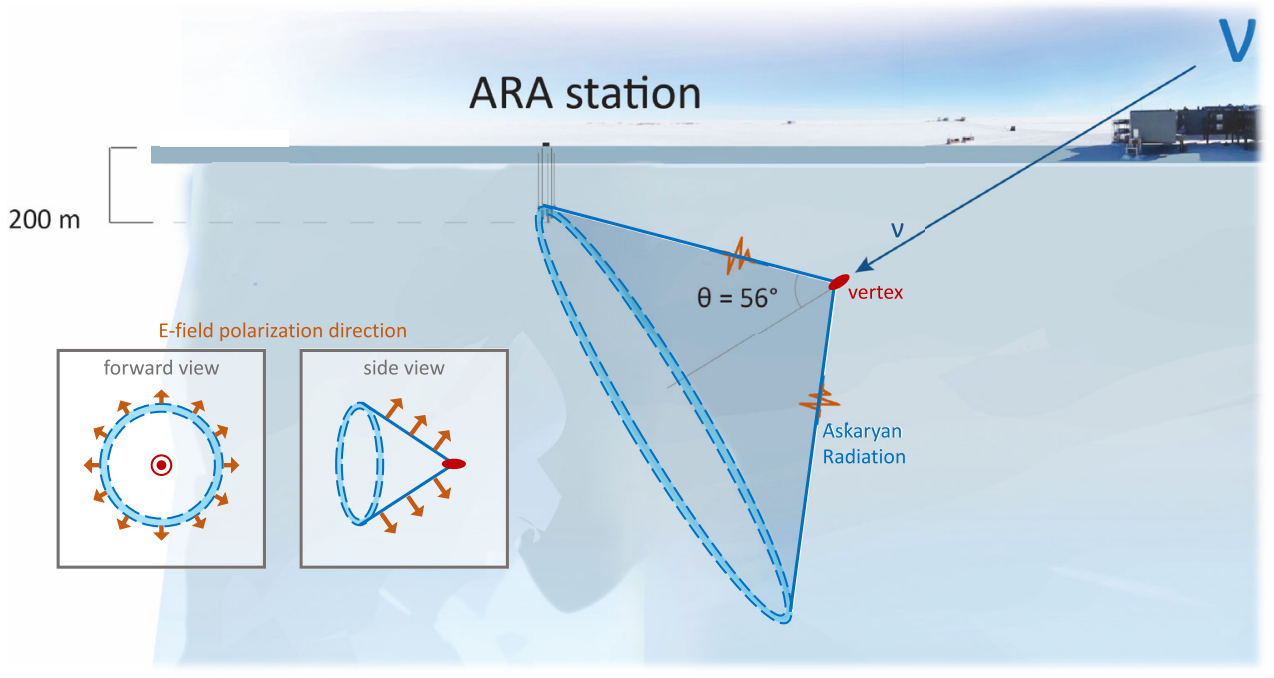

FIG. 1. A diagram showing how a high-energy neutrino interaction might be observed in an ARA station. The insets show how the Askaryan emission and its polarization would be observed if seen along, and perpendicular to, the shower axis. A more detailed view of an ARA station can be found in Fig. 2. 
neutrinos in four years of data from ARA stations A2 and A3 recorded between February 2013 and December 2016. This paper is organized as follows. In Sec. II, we describe the ARA instrument. In Sec. III, we describe the data analysis methods used in two parallel analyses, and in Sec. IV, we discuss our findings. In Sec. V, we discuss systematic uncertainties. Finally, in Sec. VI, we discuss the result and its implications, as well as prospects for the future. We also include the Appendix, where we discuss the calculation of our limit and detail the live time of the instrument.

\section{INSTRUMENT DESCRIPTION}

The Askaryan Radio Array is a UHE radio neutrino detector consisting of five stations located a few kilometers grid west of the geographic South Pole in Antarctica, as drawn in Fig. 2 [26]. A single station consists of 16 antennas, eight for detecting horizontally polarized (HPol) radiation and eight for detecting vertically polarized (VPol) radiation, along with signal conditioning and data acquisition (DAQ) electronics. The antennas are deployed at the bottom of holes at up to $200 \mathrm{~m}$ depth on four "measurement strings," forming a rectangular solid $20 \mathrm{~m}$ tall and $15 \mathrm{~m}$ deep and wide. At each corner of the rectangle, an HPol quad-slotted cylinder antenna sits a few meters above a VPol wire-frame bicone antenna. Each antenna is approximately sensitive to radiation in the $150-850 \mathrm{MHz}$ band [26]. Two "calibration strings" are deployed about $40 \mathrm{~m}$ radially away from the center of the station. Each calibration string contains a VPol and an HPol antenna, and is capable of emitting broadband rf pulses, which provide an in situ calibration of station geometry and timing, as well as a measurement of live time.
Construction of ARA began in 2011, when a prototype station (Testbed) was deployed [26,31] at $30 \mathrm{~m}$ depth to evaluate the rf environment and electronics performance. The first design station (A1) was deployed in 2012, but only up to $100 \mathrm{~m}$ depth due to limited drill performance. In 2013, two deep stations (A2, A3) that are the focus of this work were deployed at up to $200 \mathrm{~m}$ depth [32]. Two more $200 \mathrm{~m}$ depth stations (A4, A5) were deployed in 2018.

\section{A. The ARA electronics}

A schematic drawing of the ARA instrumentation and electronics is shown in the right of Fig. 2. After an incoming signal excites an antenna, it enters an antennamounted front-end signal-conditioning module; there, the signal undergoes a strong $(>50 \mathrm{~dB})$ notch filter at $450 \mathrm{MHz}$ to remove South Pole Station communications, is band-passed between 150 and $850 \mathrm{MHz}$, and boosted by approximately $40 \mathrm{~dB}$ through two stages in low low-noise amplifiers. The signal is then transmitted to the surface via rf-over-fiber to reduce attenuation over the $200 \mathrm{~m}$ journey to the top of the borehole. At the surface, the optical signal is converted back to an electronic signal, amplified again by $40 \mathrm{~dB}$, before finally being bandpass filtered once more to remove any out-of-band noise contributed by the amplifiers. The signal is then split into two paths, one for triggering and one for digitization.

The trigger path is routed through a tunnel diode which serves as a passive, few-nanosecond power integrator. When the rising edge of the tunnel diode output exceeds roughly five times the ambient thermal noise level, the lowest-level single channel trigger fires. If three samepolarization antennas register a single channel trigger within $170 \mathrm{~ns}$ (the light propagation time in the ice across
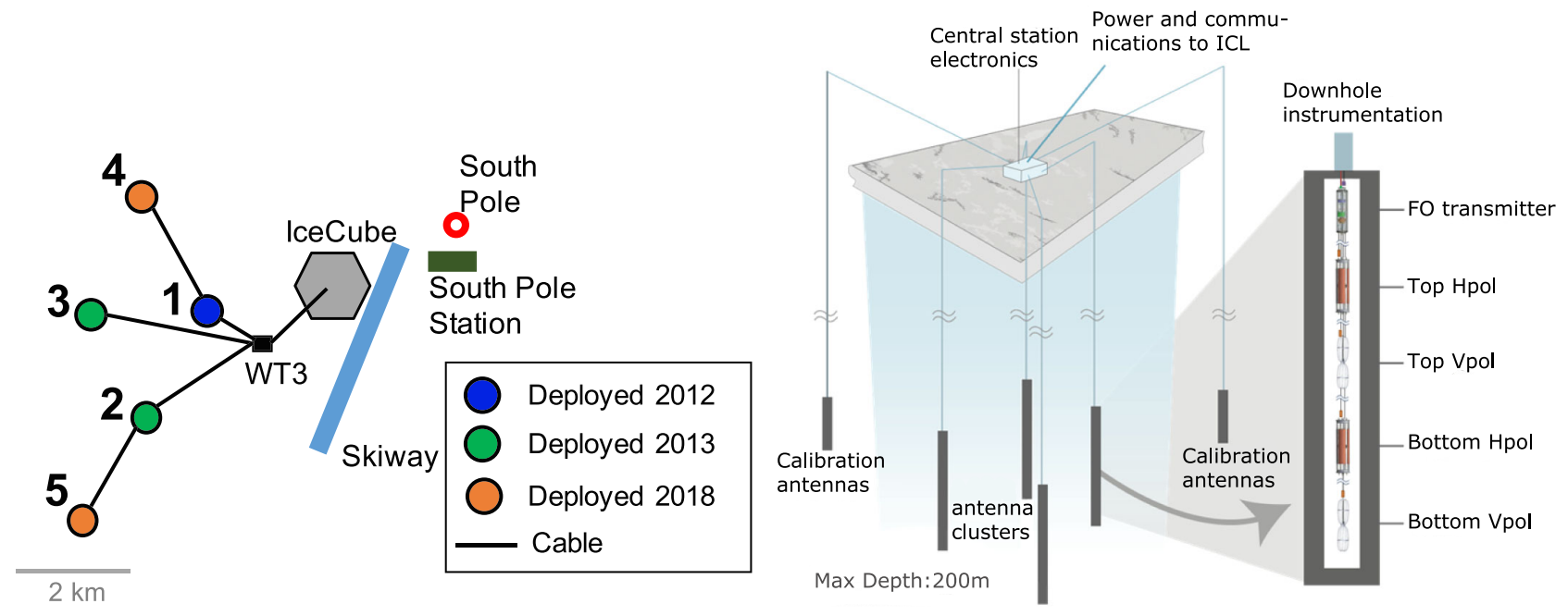

FIG. 2. (left) A top-down view of the ARA5 instrument as deployed at the South Pole, with stations color-coded by the year they were deployed. The green stations, A2 and A3, are the focus of the analysis described in this paper. (right) A schematic of the electronics and instrumentation in an ARA station; "FO" is a fiber-optic transmitter. 


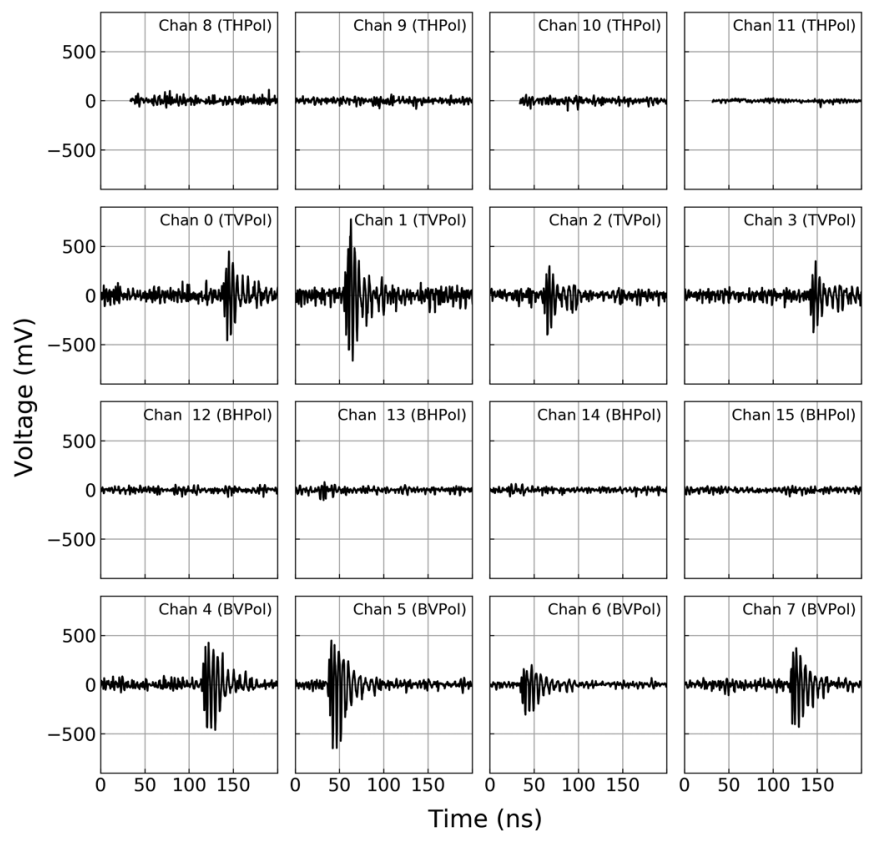

FIG. 3. An event display showing the 16 waveforms recorded in A2 for a VPol calibration pulser.

the station's diagonal), all 16 antennas in the station are read out. This scheme is optimized to trigger on Askaryan pulses, which should generate significant power in very short time windows and traverse the array at the speed of radio propagation in ice $(\sim 0.16 \mathrm{~m} / \mathrm{ns})$.

The signal is recorded through the digitization path. The signal is stored in the circular buffer of an IceRay Sampler 2 chip, which is a high-speed $3.2 \mathrm{Gs} / \mathrm{s}$ digitizer application specific integrated circuit [33]. To minimize power consumption, the buffers are implemented in analog as switched capacitor arrays $[34,35]$. After a global trigger is issued, sampling is halted and analog-to-digital conversion commences. Each readout records 400-600 ns of waveform, roughly centered on the trigger. The bundle of 16 waveforms and the associated housekeeping data (coordinated universal time timestamp, etc.) defines an event. An example VPol calibration pulser event is shown in Fig. 3, where "TVPol" notes a vertically polarized antenna deployed at the top of a string, "BHPol" notes a horizontally polarized antenna deployed at the bottom of a string, and so forth.

Triggering is performed by four Triggering DAughter boards, while digitization is handled by four Digitizing DAughter boards, with four rf channels per board. The logic and readout to storage for the eight daughter boards is managed by the ARA triggering and readout interface (ATRI). The ATRI communicates via USB with a Linux single board computer for run control and data archiving. A more detailed discussion of the ARA electronics can be found in previous work [26,32].

The precise triggering threshold for a given antenna is adjusted to maintain a single channel trigger rate for that antenna. The targeted single channel rates are chosen so that the global trigger rate, after taking into account combinatorics and trigger coincidence windows, is maintained at $5 \mathrm{~Hz}$. The dominant source of these "rf triggers" is fluctuations in the black body thermal noise background of the ice, but also includes any potential neutrino signals, as well as anthropogenic (human-made) signals such as aircraft, motor vehicles, etc. In addition, each station collects a sample of background "software" internally generated triggers as well as the calibration pulses, both at $1 \mathrm{~Hz}$, for a total $7 \mathrm{~Hz}$ global trigger rate. Every triggered event invokes approximately $1 \mathrm{~ms}$ of dead time in the electronics readout system, which has negligible $<1 \%$ impact on the live time.

\section{B. Detector live time}

This analysis comprises data recorded by ARA stations 2 and 3 (A2 and A3) between initial deployment in February 2013 and the end of December 2016. Over the course of these four years, each station accumulated roughly 1100 days of live time, as shown in Fig 4, recording over 1.2 billion events in total between the two stations. The two detectors were operated in several different "configurations," representing different combinations of operating parameters such as trigger window size, etc. We summarize the five data taking configurations for each station in Table II of the Appendix. For all configurations in A2, the bottom HPol channel of string 4 was nonoperational,
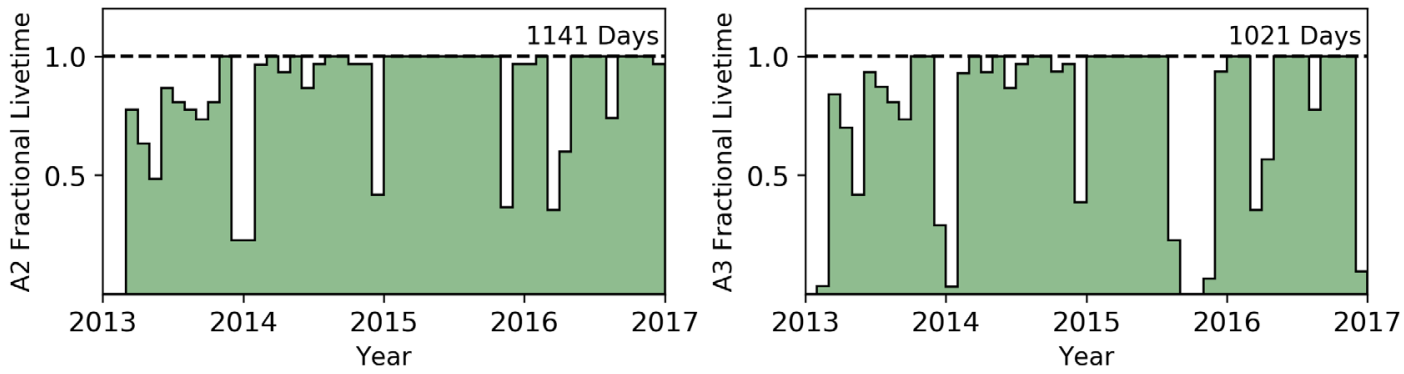

FIG. 4. Operational fractional live times for A2 (left) and A3 (right) from deployment in February 2013 through the end of the analysis period in 2016; each bin is one month wide. From the four years of deployment, 1141 days from A2, and 1021 days from A3, are good for analysis. This is mostly due to intermittent downtime; quality cuts remove less than $2 \%$ of live time. 
and it is excluded from participating in the trigger for configurations 3-5. Additionally, for configurations 3-5 of A3, the fourth string of the detector participates in forming triggers normally, but due to technical problems in the digitization chain it does not produce useful signal for analysis.

\section{Simulation}

We generate simulated data sets with the Monte Carlo package AraSim, which has been previously described extensively in Allison et al. [31,32]. This code models the generation of neutrino events from a diffuse flux and their interactions with Earth and Antarctica. After simulating interactions in-ice, AraSim renders a time-domain parametrization of the Askaryan radiation and propagates that radiation through the ice, taking into account signal attenuation and ray bending based on a depth-dependent index of refraction model. When the radiation arrives at a simulated station, it is convolved with a frequency-dependent model of the detector, including the antennas, signal chain, and the trigger logic. The model of the instrument includes the dispersive effect of the signal chain that induces a frequency-dependent group delay. If the event satisfies a simulated trigger, it is stored in the same format as real data so that our analysis codes can be executed on either data or simulated events interchangeably.

The models of the A2 and A 3 stations are data driven and include calibrations derived from the 2012 to 2013 data set

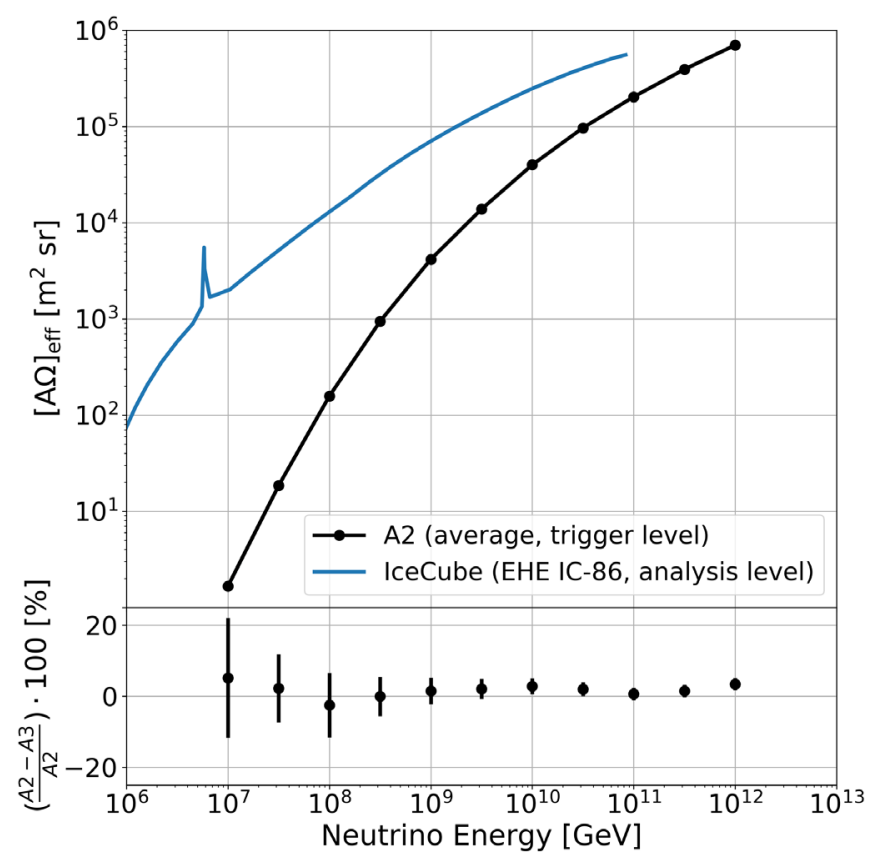

FIG. 5. (top) The simulated trigger-level effective area steradian $\left([A \Omega]_{\mathrm{eff}}\right)$ for $\mathrm{A} 2$, averaged across configurations. For comparison, we also show the analysis-level sensitivity of IceCube [36]. (bottom) The percent difference between the A2 and A3 effective areas. as described in [32]. In particular, the antenna locations, the noise temperature of the ice, and the gain of every channel are all implemented in the model based on in situ measurements. The simulation also models the configurationspecific variations in the electronics behavior (readout length, trigger window size, trigger delay values, etc.) as detailed in the Appendix.

In Fig. 5, we show the aperture $\left([A \Omega]_{\text {eff }}\right)$ of $\mathrm{A} 2$, averaged over configurations. The effective area is derived via Monte Carlo techniques with AraSim as described in the Appendix. For comparison, we also plot the effective area of the IceCube experiment [36]. As can be seen in the bottom panel, we find that $\mathrm{A} 2$ and $\mathrm{A} 3$ have comparable effective areas to within a few percent. We additionally find that triggering and readout parameters specific to each live time configuration, as discussed in Sec. II B, do not result in differences in the trigger level effective area in excess of a few percent. The two detectors, A2 and A3, are simulated independently; previous studies have shown that only a small fraction of events trigger both $\mathrm{A} 2$ and $\mathrm{A} 3$ simultaneously, amounting to about $5 \%$ of events at $1 \mathrm{EeV}$ [32].

\section{DATA ANALYSIS}

Our data analysis searches for a diffuse flux of neutrinos between $10^{16}$ and $10^{21} \mathrm{eV}$. The analysis is designed to remove background events, principally thermal and anthropogenic noise, while preserving sensitivity to neutrinos. The analysis proceeds in a "blind" fashion, where the ARA data are divided into two subsets. A "burn" sample of $10 \%$ of the data, which is assumed to be representative of the full data sample, is set aside and used to tune cuts and understand backgrounds. The remaining $90 \%$ of the data are kept blinded until cuts are finalized. Before unblinding, it was decided that in the absence of a detection, the analysis with the best expected limit would be used to set the limit.

\section{A. Summary of blind analyses}

Two parallel, complementary analyses were performed on the four-year data samples, which we refer to as Analysis A and Analysis B. In this section, we outline the strategies followed by both, with Sec. III F describing features specific to the two separate analyses.

Both analyses follow similar strategies. First, a set of basic data quality and live time cuts are applied to remove detector "glitches," calibration events, and periods of live time known to be contaminated with anthropogenic activities. Second, fast event-level filters designed to reduce the quantity of data by an order of magnitude or more are applied. Third, interferometric-based reconstruction is performed to identify the arrival direction of a recorded signal and geometric cuts invoked to reject events that originate from above the ice surface or in the direction of known calibration pulsers. Finally, a bivariate cut is applied on the 
signal strength and a reconstruction quality parameter. Analysis A considers events only in the vertical polarization, while Analysis B includes events in both the horizontal and vertical polarizations. Both derive data-driven models of the background, and set their final cuts such that $\sim 0.01$ background events are expected to pass the analysis in the 1100 days of live time, corresponding to the level at which we find the best expected limit.

Both analyses use the $10 \%$ burn sample to tune cuts and understand backgrounds. The number of neutrinos expected in the burn sample based on allowed models is $<0.02$, so the chance of excluding a neutrino candidate in the burn sample is negligible. Moreover, cuts were motivated by an optimization procedure and by examining outlying events that did not have neutrinolike properties, not by the objective to eliminate all events in the burn sample. The distribution of the background was smooth and followed a statistical distribution, and we did not readjust cuts to remove specific neutrinolike events.

\section{B. Data quality cuts}

Before analysis begins, we remove periods of live time which are known to contain human and calibration activity. This includes, for example, maintenance operations on the detector during the Austral summer, and the operation of surface pulsers or pulsers deployed on two of the final IceCube strings (strings 1 and 22), known as the "IceCube Deep Radio Pulsers." These live time cuts remove less than $2 \%$ of the total live time recorded by the instrument.

Next, both analyses deploy a nearly common set of data quality cuts designed to remove instrumental glitches and remaining calibration events from the data set. Glitches are typically present either as waveforms that are shorter than those generated during normal readout, or waveforms with unphysical discontinuities (likely due to digital errors in the readout electronics), and comprise less than $0.001 \%$ of events. Additionally, we remove the internally generated software triggers described above in Sec. II A, as well as "tagged" calibration pulser events. We are able to tag calibration pulsers under normal operating conditions as they are nominally triggered by the pulse-per-second (PPS) TTL inside the DAQ, so these events are readily identified by their timestamps. This has negligible effect on the detector live time and neutrino sensitivity. The quality cuts that are not common between analyses focus on slightly different methods for detecting unphysical discontinuities in the waveforms, as well as the identification of out-ofband power content.

\section{Event filter}

Because of the large size of the ARA data set $\left(>1.5 \times 10^{8}\right.$ events/station/year), and the expectation that most triggers are upward fluctuations of the thermal noise environment, each analysis applies a computationally simple cut that rejects $>90 \%$ of triggered events.
Analysis A utilizes an event filter based on a multiplicity condition, which requires that more than three VPol channels each has a signal strength above a threshold. Analysis B utilizes a wave-front-rms filter, which requires that the pattern of arrival times across the array be consistent with that of a plane wave. Both algorithms have been described elsewhere [37]. Analysis A tunes its filter such that $99 \%$ of triggered events do not pass the filter, while Analysis B tunes its filter such that approximately 90\% of triggered events do not pass. In Analysis A, the signal strength threshold is tuned. In Analysis B, the signal strength threshold and tolerance parameter for deviation from plane wavelike timing are tuned. In Analysis A, the multiplicity trigger is approximately $70 \%$ efficient for $10^{18} \mathrm{eV}$ neutrinos, where for Analysis B the wave-frontrms filter efficiency is approximately $90 \%$.

\section{Reconstruction and geometric cuts}

For events passing the event filter, we perform an interferometric-based reconstruction to determine the direction of the source of measured incoming radio waves. This interferometric reconstruction technique has been used in other ARA analyses $[31,32,38,39]$ and in the ANITA experiment [40]. The interferometric technique relies on the relationship between the location of an emitting source in space and the time delays expected for two measurement antennas with known separation.

For a given pair of antenna waveforms, the crosscorrelation $C_{\mathrm{i}, \mathrm{j}}$ between the voltage waveform on the $i$ th antenna $\left(V_{\mathrm{i}}\right)$ and the voltage waveform on the $j$ th antenna $\left(V_{\mathrm{j}}\right)$ as a function of time lag $\tau$ can be expressed in Eq. (1),

$$
C_{i, j}(\tau)=\frac{\sum_{t} V_{i}(t) V_{j}(t+\tau)}{\mathrm{rms}_{i} \times \mathrm{rms}_{j}},
$$

where rms is the root-mean-square voltage of the waveforms in the absence of signal. The lag $\tau$ defines the time delay of one antenna waveform relative to the other and depends on the position of the source emitter relative to the array center, characterized by an elevation angle $(\theta)$, an azimuthal angle $(\phi)$, and a distance to the source $(R)$. The array center is defined as the centroid of all 16 measurement antennas in the station.

The pairwise time lags $\tau$ for a given point on the sky $\theta, \phi$ are computed by calculating the path a light ray would take from a hypothesized source located at a distance $R$ to an antenna. The calculation accounts for the changing index of refraction of the Antarctic firn, which causes rays to follow curved, rather than rectilinear trajectories. With $n(z)$ the depth-dependent index of refraction, and $z$ the (negative) depth from the ice surface, the ray-tracing method models the changing index of refraction as

$$
n(z)=1.78-1.35 e^{0.0132 z} .
$$


This index of refraction model was determined by fitting data collected by the RICE experiment in Antarctica [41]. We consider the index to be unity above the surface.

The total cross-correlation strength for a given point on the sky is given by summing over all like-polarization pairs of antennas as in Eq. (3),

$$
C_{\text {sky }}(\theta, \phi ; R)=\frac{\sum_{i=1}^{n_{\text {ant }}-1} \sum_{j=i+1}^{n_{\text {ant }}} C_{i, j}[\tau(\theta, \phi ; R)]}{n_{\text {ant }}} .
$$

To smooth uncertainties in the ice model and other systematics (such as differences in the phase responses of the various contributing antennas), we calculate the Hilbert envelope of the cross-correlation function before summing over pairs, as is done in previous analyses. The Hilbert envelope of the cross-correlation $H\left(C_{i, j}\right)$ is calculated according to Eq. (4),

$$
H\left(C_{i, j}\right)=\sqrt{C_{i, j}^{2}+h^{2}\left(C_{i, j}\right)},
$$

where $h\left(C_{i, j}\right)$ denotes the Hilbert transform.

The cross-correlation function for an individual pair of antennas, $C_{i, j}$, is expected to be maximal when the lag is equal to the true difference in the arrival times of a signal at the two different antennas. The sky map is therefore expected to have a peak at the putative source direction.

For determining source direction, Analysis A tests radii from 40 to $5000 \mathrm{~m}$ to locate a hypothesis radius which maximizes $C_{\text {sky }}$, while Analysis B reconstructs only at 41 and $300 \mathrm{~m}$, corresponding to the radius of the calibration pulser and a radius taken as a plane-wave proxy. That one analysis performs a radius scan is a setup inherited from a separate investigation regarding our ability to determine the radius of curvature for signals, which we found to be limited for sources beyond a few hundred meters, given the instrumental timing resolution. After finding the best reconstruction direction (the direction which maximizes $C_{\text {sky }}$ ), both analyses impose two geometric cuts. The first is an angular cut in the direction of the calibration pulser system. The second is a cut on the reconstructed elevation $(\theta)$ of the hypothetical source relative to the station center and is used to reject events coming from above the surface.

The cuts on the angular region around the calibration pulser systems is necessary to reject untagged calibration pulser events; approximately 1 in $10^{4}$ calibration pulser signals is emitted outside the time window expected; the cause of this "misfiring" is not well understood. Additionally, one configuration in A3 (configuration 2) did not have the calibration pulser system correctly synchronized to the PPS clock, and so a purely geometric rejection criterion is needed. To determine this geometric cut region, the angular distribution of tagged calibration pulsers is fit (either with a Gaussian or a Kernel density estimator) and a cut region determined such that fewer than $10^{-3}$ calibration pulser events are expected to reconstruct outside of that angular region for the entire live time period. The angular cut region is an approximately $10^{\circ} \times 10^{\circ}$ box around the true calibration pulser location. The value of $10^{-3}$ is approximately an order of magnitude less than the number of background events expected to pass all analysis cuts. Less than $3 \%$ of neutrinos are cut by this calibration pulser geometric cut requirement.

The geometric cut at the surface is used primarily to reject anthropogenic noise, as well as potential downgoing physics signals such as cosmic rays. We make the cut on events from above the surface because we expect neutrino events to predominantly yield upcoming signals. The cut on events from above the surface proceeds similarly to the calibration pulser geometric cut. We fit the distribution of events in $\sin (\theta)$ near the transition between the air-ice boundary and place an angular cut such that fewer than $10^{-3}$ events from the above-ice distribution are expected to reconstruct within the ice. In Analysis A, events are only reconstructed in the vertical polarization, while in Analysis $\mathrm{B}$, an event may be classified as having an above-thesurface origin in either polarization, and if so it is rejected from consideration in the searches in either polarization. The cut on the reconstruction angle $\theta$ varies from $11-35^{\circ}$, and approximately $10 \%-30 \%$ of neutrinos are cut by the surface cut at $10^{18} \mathrm{eV}$, depending on the analysis, station, and configuration. For example, in Analysis A, the angular cut is $\sim 30^{\circ}$ for $\mathrm{A} 2$, but is $\sim 10^{\circ}$ for $\mathrm{A} 3$. The reduction in efficiency is partially because radio waves can follow curved trajectories as they traverse the varying index-ofrefraction and can appear as downgoing signals when they in fact arise from sources within the ice.

\section{E. Bivariate cut and background estimate}

Both analyses implement their final separation of noise from potential neutrino signals as a bivariate cut in the peak cross-correlation $\left(C_{\text {sky }}\right)$ vs signal strength $(\Gamma)$ plane. For an event to "pass," Analysis A imposes a box cut requiring that an event's $C_{\text {sky }}$ and $\Gamma$ both exceed a station and configuration-specific threshold: $C_{\text {sky }}>C_{\min }$ and $\Gamma>\Gamma_{\min }$. In Analysis B, an event is required to pass a linear combination of the two, such that $\Gamma>m C_{\text {sky }}+b$, where $m$ and $b$ are station- and configuration-specific analysis parameters. An example of the box cut for A3 configuration 3, in Analysis A, is provided in Fig. 6.

For the purpose of Fig. 6, we show $\Gamma$ in the way it was computed to perform cuts in Analysis A. This definition of signal strength we call the root power ratio (RPR), and is defined as $\mathrm{RPR}=E_{j, \text { max }} / \sigma_{E_{j, \text { noise }}}$, where $E_{j, \max }$ is the maximum of the square root of a rolling $25 \mathrm{~ns}$ integrated power average of the waveform, specifically 
A3 Config 3 Data

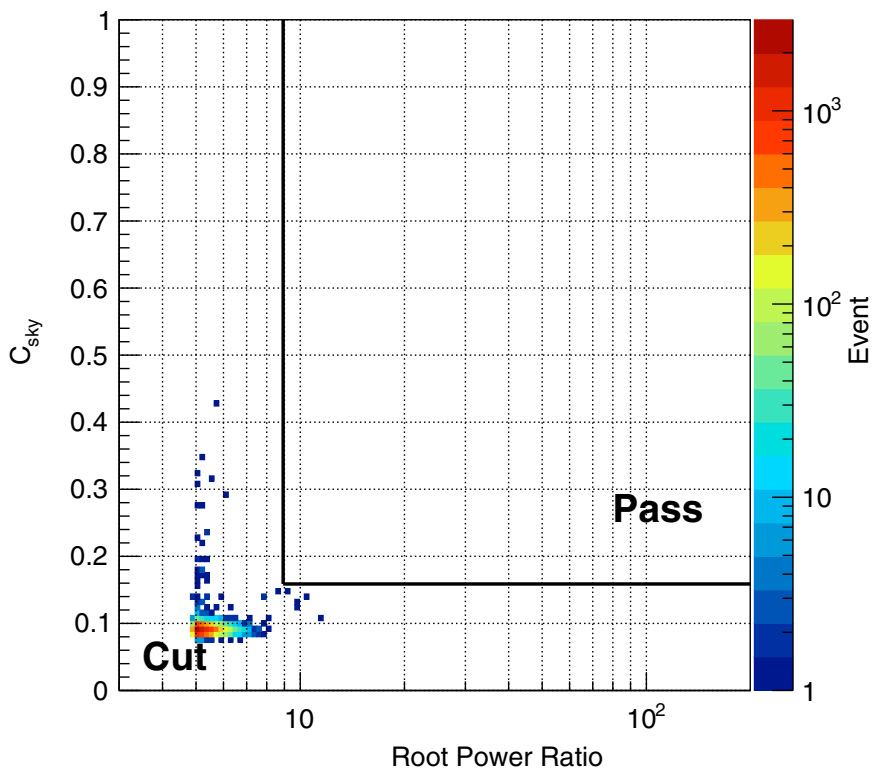

A3 Config 3 Simulation Ev=1 EeV

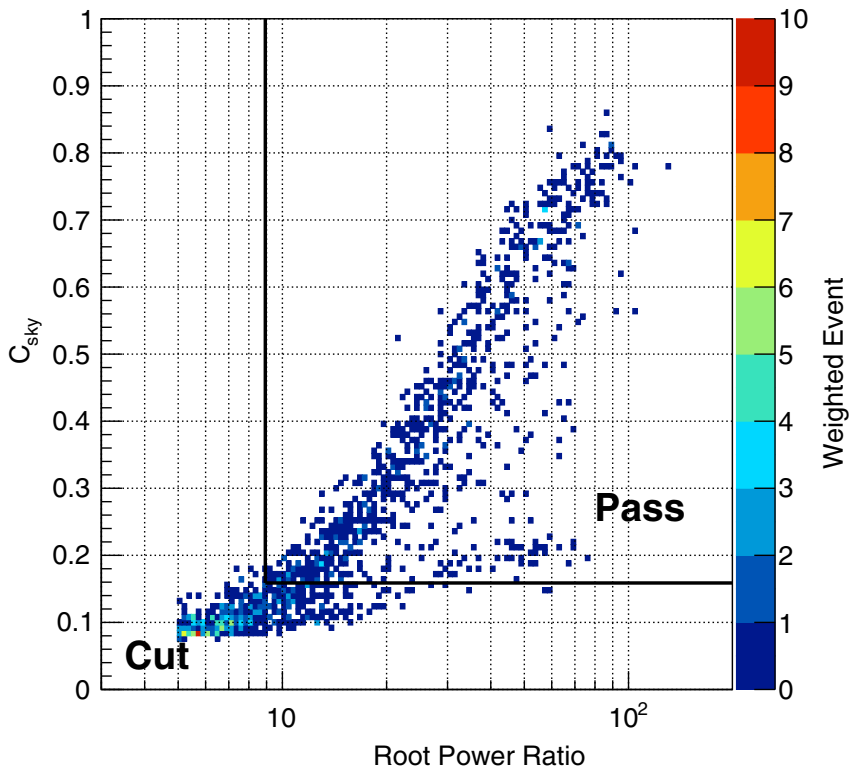

FIG. 6. An example of the bivariate cut plane, for which the final 2D box cut is made for A3 configuration 3. (left) The plane as observed in 10\% "burn-sample" data, showing events clustering at low correlation and low root power ratio. (right) The plane populated with simulated neutrinos at $10^{18} \mathrm{eV}$, showing events distributed throughout. Events at low correlation and low root power ratio are cut; events at higher values define the signal region and pass the analysis.

$$
E_{j}=\sqrt{\frac{1}{n} \sum_{i=j}^{j+n} V_{i}^{2}},
$$

where $n$ is the number of samples in the $25 \mathrm{~ns}$ window and $\sigma_{E_{j, \text { nose }}}$ is the rms value of $E_{j}$ in the half of the waveform that does not contain the maximum. This RPR variable has been used in a previous ARA analysis [32] and was chosen to more closely emulate the power-integrated envelope that is used in the ARA trigger.

Both analyses use a data-driven model of the backgrounds in order to set final cuts and estimate the expected number of background events passing all cuts. As in previous analyses, the model is constructed by fitting the distribution of events as a function of the cut parameters $\left(C_{\text {sky }}, \mathrm{RPR}\right.$, etc.), and setting the cut such that fewer than $\sim 0.01$ background events are expected to pass all cuts, which is the level at which we find the best expected limit based on statistical uncertainties only.

Before examining the neutrino signal region, defined as events passing all cuts in the analysis, both analyses first reversed the requirement that events reconstruct inside the ice. That is, we examined events which failed the geometric cut by reconstructing to the surface. This is done in order to identify bursts of activity from the surface, and we exclude runs which have $\gtrsim 11$ events reconstructing to the surface. At this stage, we do not exclude single, isolated events, "singlets," as neutrinos are expected to arrive isolated in time and space. In both analyses, this "surface-noisy" cut eliminated approximately an additional week of live time.

\section{F. Analysis-specific comments}

\section{Analysis A}

Analysis A uses solely signal from VPol antennas for the search. This is motivated by the fact that the majority $(\sim 70 \%)$ of simulated signal events contain VPol triggers. This is partly because VPol antennas are more sensitive than HPols antennas, especially at low frequencies. To define the surface geometric cut, Analysis A reconstructs the incident angle of each event with signal arrival times calculated assuming a bulk-ice model with a constant index of refraction 1.76 and a putative source distance of $5 \mathrm{~km}$ to emulate a distant source at the ice surface. This approach proved to be the most successful in reconstructing a radio emitter system installed on the rooftop of the IceCube Lab, which served as a proxy for distant surface signals. The cut is then placed on the elevation angle of the result of this reconstruction as described in Sec. III D.

One category of background present in ARA data is continuous-wave (CW) emission. $\mathrm{CW}$ emission is anthropogenic in origin and presents as a strong spectral peak in the power spectral density of an event. The most common type of $\mathrm{CW}$ encountered in ARA is generated by the $\sim 403 \mathrm{MHz}$ radiosonde attached to National Oceanic and Atmospheric Administration weather balloons that are launched once or twice daily from the South Pole; we additionally see $125 \mathrm{MHz}$ emission from an as-yet unidentified source.

To eliminate the contamination of $\mathrm{CW}$ emission, Analysis A places an out-of-band cut, where an event is 
considered CW contaminated if more than three channels in either polarization demonstrate peak spectral density below $170 \mathrm{MHz}$. This frequency threshold is motivated by the edge of the pass-band filter. We discard the event entirely if such CW contamination is found. This cut represents negligible signal efficiency loss below $10^{19} \mathrm{eV}$ and a $\sim 10 \%$ loss at $10^{21} \mathrm{eV}$ from off-cone signals. To reject $\mathrm{CW}$ contamination in higher frequencies, we observe that such events, while producing high $C_{\text {sky }}$ values due to their CW nature, do not produce high RPR values on the $C_{\text {sky }}{ }^{-}$ RPR plane. Therefore, Analysis A rejects these events with the $2 \mathrm{D}$ box cut.

\section{Analysis B}

Analysis B features two major differences from Analysis A. First, Analysis B performs the neutrino search in both polarizations, VPol and HPol. Second, Analysis B filters power in events with CW contamination. CW contamination is identified with two methods: first by looking for spectral peaks over run-specific baselines as in the prototype station analysis [31] and second by looking for stability between phasors at a given frequency as is done in the LOFAR experiment [42]. Once $\mathrm{CW}$ has been identified at a specific frequency, this contamination is removed using a filtering technique developed and used by the ANITA Collaboration which operates in a similar frequency domain $[43,44]$. The filter notches spectral peaks in the amplitude domain, while reconstructing the phasors representing the signal and thermal noise contributions only, with CW contamination removed. Once an event has been filtered of its contaminating $\mathrm{CW}$ emission, it proceeds in the analysis as above.

Development and use of techniques to mitigate $\mathrm{CW}$ contamination is important because the $\sim 403 \mathrm{MHz}$ emission at South Pole can contaminate up to $10 \%$ of ARA's daily live time. As the detectors continue to accrue live time, and sensitivity to weak signals improves, the ability to filter events of contaminating $\mathrm{CW}$ emission will be important for leveraging the full live time of the array.

\section{RESULTS}

After rejecting data containing bursts of surface activity, both analyses examined the neutrino signal region in A2 before examining the signal region in A3. Each analyses' individual unblinding results are discussed below in Secs. IV A and IV B.

Neither analysis observes a statistically significant excess of events, observing zero events against the estimated background. In the absence of detection, in Fig. 7, we compute the $90 \%$ confidence level (CL) upper limit on the diffuse flux of neutrinos.

Further details on the upper limit calculation, including inclusion of the systematic uncertainties discussed in Sec. V, can be found in the Appendix. Inclusion of the

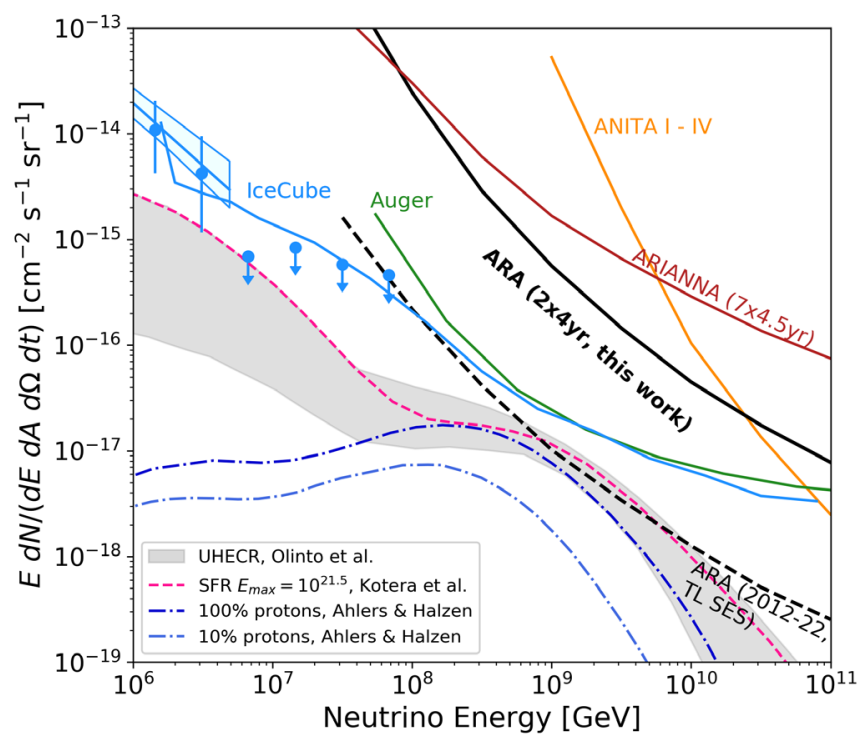

FIG. 7. The $90 \%$ confidence-level upper limit on the all-flavor diffuse flux of neutrinos set by this analysis (thick black line). The limit accounts for uncertainties in the background estimate and systematic uncertainties on the neutrino sensitivity. We also plot the projected trigger-level single-event sensitivity (TL SES) for the five-station ARA5 array by 2022 as a black-dashed curve. Also shown are the latest limits and flux measurements from IceCube [20,45], Auger [21] (rescaled with decade-wide bins and for all-flavors), ANITA [23] (rescaled with decade-wide bins), and ARIANNA [24]. Shown for comparison are several benchmark cosmogenic neutrino flux models $[13,16,50]$.

systematic uncertainties in the limit has an $\mathcal{O}(5 \%)$ effect. We report the limit set by Analysis A, which had slightly superior expected sensitivity, by up to $15 \%$, depending on energy. As a benchmark, the number of events expected to be observed in the analysis ranges from 0.25 for an unbroken extrapolation of the astrophysical neutrino flux as measured by IceCube with a spectral index of -2.13 [45], to 0.027 in the case of a cosmogenic neutrino flux where protons make up only $10 \%$ of cosmic ray primaries [16].

In Fig. 8, we present the analysis efficiency of Analysis A for both A2 and A3; we plot the average signal efficiency, taking into account the variations due to different run configurations and their respective live times. The signal efficiency is calculated by simulating neutrinos in AraSim and taking the ratio of the number of neutrinos passing the analysis cuts to the number of neutrinos that trigger the detector. We show the efficiency for Analysis A, as it is the analysis used to set our limit, though the efficiencies for Analysis B (which was developed in a parallel and independent fashion) are comparable. On the left, we show the efficiency as a function of SNR, where SNR is computed as the third highest $V_{\text {peak }} / \mathrm{rms}$, where $V_{\text {peak }}$ is the highest absolute voltage peak in a waveform, and the rms is the root-mean-square of the voltage values in 

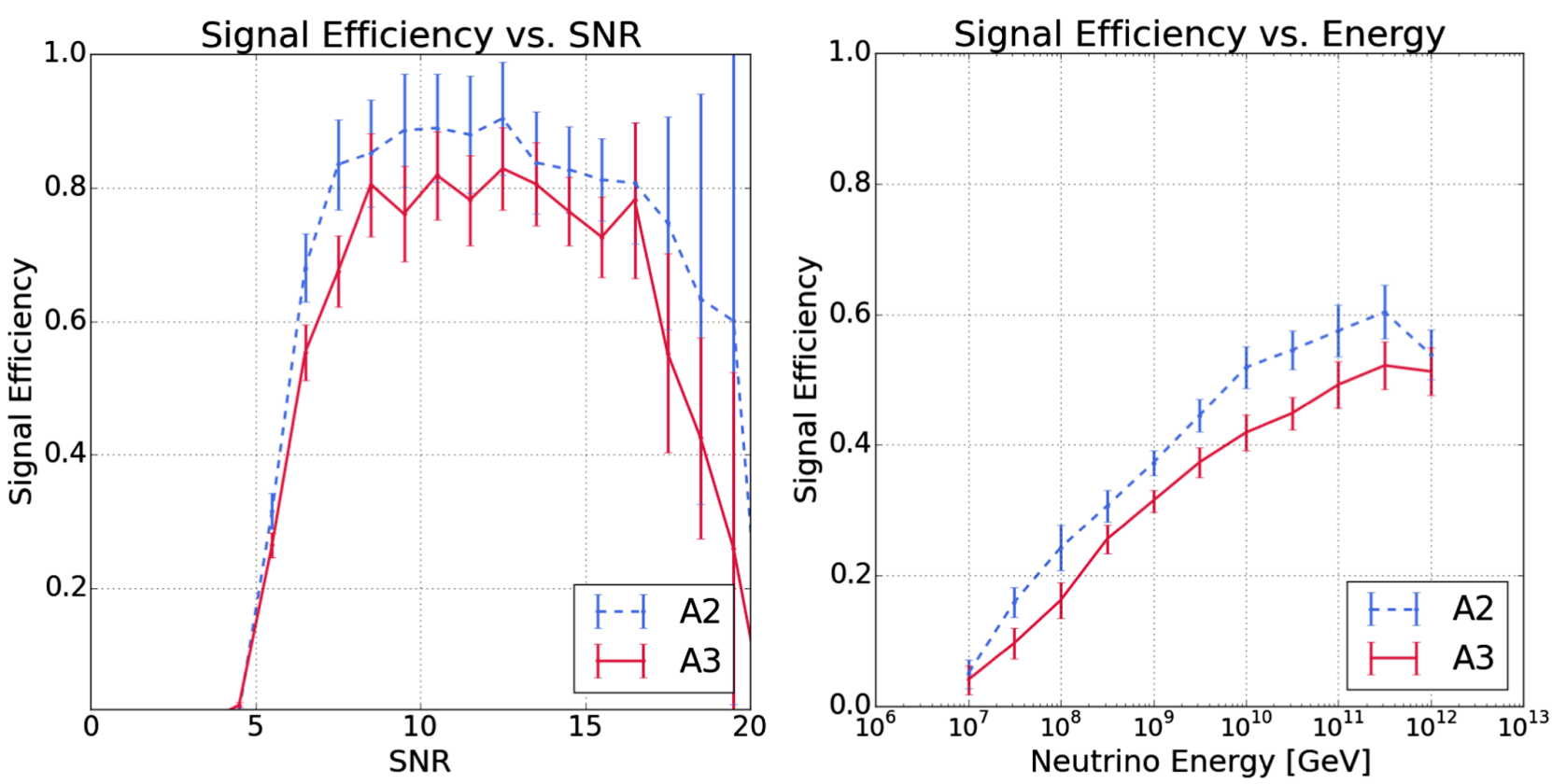

FIG. 8. Monte Carlo estimated analysis efficiency as a function of signal-to-noise ratio (left) and neutrino energy (right) for Analysis A. For context, the trigger efficiency of an ARA station has been measured to reach 50\% at an SNR of 3.7 [46]. In the left figure, we assume an unbroken power-law spectrum with a spectral index of -2.13 to weight the energies contributing to the efficiency. The efficiency decrease around SNR $=14$ is due to waveform saturation effects as simulated in Arasim.

that waveform. We present the figure with this definition of SNR as it more closely aligns with that commonly used for comparison purposes in the literature. The analysis becomes efficient near an SNR of 6 and does not fully saturate to a value between $75 \%$ and $90 \%$ until it is above an SNR of 8 . The saturated efficiency for A3 is $~ 10 \%$ lower than for $\mathrm{A} 2$ because $\mathrm{A} 3$ required a larger angular cut region to reject surface events, as discussed in Sec. III D. On the right, we show the efficiency as a function of energy. At $10^{16} \mathrm{eV}$, the analysis has a relatively low efficiency of about $5 \%$. The efficiency rises to $\sim 35 \%$ by $10^{18} \mathrm{eV}$ and peaks near $10^{20} \mathrm{eV}$ at between $50 \%$ and $60 \%$, depending on the specific station. Efficiencies for all stations and configurations are provided in additional figures in the Appendix.

\section{A. Analysis A results}

After post-unblinding examination, Analysis A observes 0 events on a background expectation of $(5 \pm 2) \times 10^{-2}$ background events per station.

At unblinding, Analysis A observed two events in the candidate neutrino signal region in A2. While both reconstruct inside the ice using an interferometric technique which utilizes all VPol channels of the array, both only have visibly identifiable signals in the bottom row of VPol antennas. When the reconstruction is repeated utilizing only antennas where the signal strength exceeds the event filter threshold, both events confidently reconstruct to above the surface. We consider both of these events to be backgrounds of surface origin.

At unblinding, Analysis A observed four events in the candidate neutrino signal region in A3. Three cluster in time to within a few minutes, and are located in a run which contains a burst of surface noise, but was technically subthreshold in the surface-noisy cut as described above in Sec. IV. The fourth event is reconstructed inside the ice when all VPol channels participate in the interferometry. Again, if only channels with signal strength above the event filter threshold are considered, then the event reconstructs to above the surface. It is therefore determined to be consistent with a background of surface origin.

Since all events observed in Analysis A can, with currently available tools, be identified to be of surface origin, or cluster in time with bursts of surface activity, we do not consider Analysis A to have measured any events. The post-unblinding cut necessary to remove the misreconstructed surface events results in a negligible efficiency loss $(\leq 0.25 \%)$. As Analysis A provided the better expected limit, we proceed to compute the limit as described in the Appendix with a observed number of events of zero.

\section{B. Analysis B results}

After post-unblinding examination, Analysis B observes 0 events on a background expectation of $(1 \pm 0.3) \times 10^{-2}$ events per station.

At unblinding, Analysis B observed 19 events in the candidate neutrino signal region in $\mathrm{A} 2$. Of these, seven 
were "near-surface" events, and were addressed by more stringent, data-driven surface cuts, as described in Sec. III D. Analysis B had originally used a geometric argument to determine the value of the surface cut, as opposed to data-driven methods. An additional seven events were of a type not observed in the burn sample, where an unphysical amount of power was deposited in one or two strings. These were removed with an update to the quality cuts, and the update had negligible impact on the signal efficiency. One event was a calibration pulser that misfired during a time when it was not enabled by the software. It was misreconstructed in the $41 \mathrm{~m}$ interferometry, but was correctly reconstructed in the $300 \mathrm{~m}$ radius, and was removed by additionally rejecting events if they reconstructed towards the calibration pulser in either interferometric radii. This additional calibration pulser geometric rejection also had negligible impact on the analysis efficiency. To address the remaining four events, an additional hit-time-based reconstruction method, which traces its lineage to the RICE experiment, was added. The method uses a integrated-power envelope [the same as described in the definition of RPR in Eq. (5)] to identify hit times in the waveforms and requires at least four waveforms in the event to have an RPR above a threshold of eight. The method then searches for the location of a source emitter $(\theta, \phi, R)$ which minimizes the differences between the predicted and observed time delays between channels. With this additional cut, all four of the remaining events in Analysis B are rejected - one fails to have enough hits to be reconstructed and the remaining three reconstruct to above the surface.

At unblinding, Analysis B observed three events in the candidate neutrino signal region in A3. Two cluster in time within a few minutes and are located in the same run which generated the three passing events in Analysis A. The third is the same event observed in Analysis A, which was determined to be downgoing both in Analysis A through the revised interferometric method described above, and also in Analysis B independently with the hit-time-based reconstruction method. Like in Analysis A, all three events are determined to be of surface origin, or associated with a burst of surface activity.

Since all events observed in Analysis B can, with currently available tools, be identified to be of surface origin, or cluster in time with bursts of surface activity, we do not consider Analysis B to have measured any events. The additional postunblinding hit-time-based reconstruction cut results in no more than an additional $2 \%$ efficiency loss.

\section{SYSTEMATIC UNCERTAINTIES}

In this section, we describe the systematic uncertainties considered in the analysis. The impact of these systematics on $[A \Omega]_{\text {eff }}$ are shown in Fig. 9, and a table summarizing the magnitude of their effects at $10^{18} \mathrm{eV}$ is provided in Table I. We consider systematic uncertainties broadly in two classes. The first class is associated with theoretical

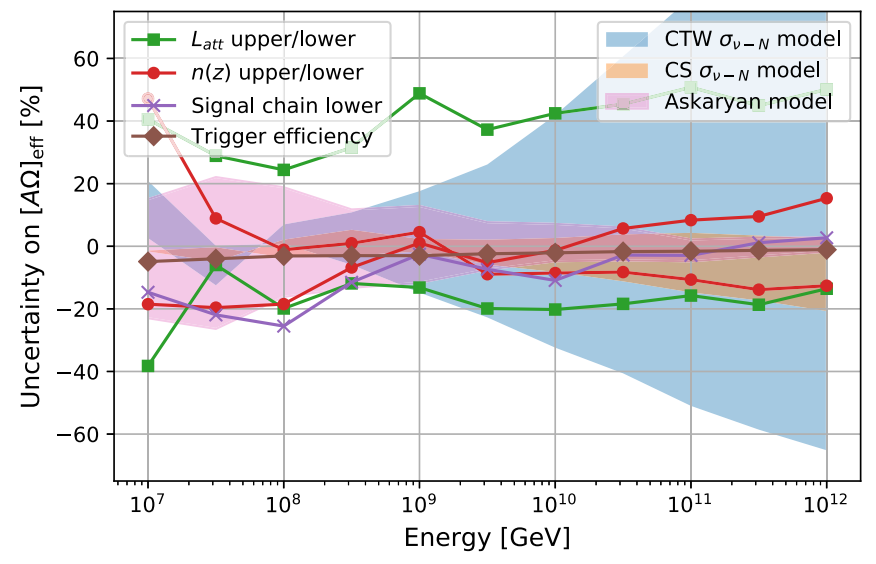

FIG. 9. Uncertainties between the central values used in the simulation and upper/lower bounds for each model parameters. Theoretical systematics (shaded regions), such as the Askaryan model and the neutrino-nucleon cross section, are not accounted for when calculating the neutrino limit. Uncertainties associated with the detector and medium (dashed and solid lines) are accounted for in the calculation.

uncertainties surrounding the neutrino-nucleon cross section and Askaryan emission, and are shown in Fig. 9 as solid bands, reported at the trigger level. The second class is associated with uncertainties in our understanding of the detection medium and our instrument. The latter are taken into account in setting the final limit as described in the Appendix and are shown as dashed/dotted lines in Fig. 9 at the analysis level.

For the neutrino-nucleon cross section $\left(\sigma_{\nu-N}\right)$, AraSim uses the model derived by Connolly, Thorne, and Waters (CTW) [18]. The upper and lower bounds for $\sigma_{\nu-N}$ are substituted for the central value in the simulation to estimate the effect of the uncertainty on the simulated $[A \Omega]_{\text {eff }}$ at the trigger level. In the CTW model, the uncertainties on $\sigma_{\nu-N}$ are large and grow as a function of energy, exceeding $100 \%$ above $10^{21} \mathrm{eV}$. At $10^{18} \mathrm{eV}$, the uncertainties on the trigger-level effective area due to the cross section are estimated at $-15 \% /+18 \%$. In Fig. 9, for comparison, we also show the uncertainties if we use an alternative cross section developed by Cooper-Sarkar et al. (CS) [19] which has smaller uncertainties at high energies

TABLE I. A summary of the systematic uncertainties in the neutrino sensitivity at a neutrino energy of $10^{18} \mathrm{eV}$.

\begin{tabular}{lcc}
\hline \hline Systematic uncertainty & $+(\%)$ & $-(\%)$ \\
\hline Cross section (CTW) & 18 & 15 \\
Askaryan emission & 13 & 11 \\
Attenuation length & 50 & 8 \\
Index of refraction & 5 & 5 \\
Signal chain & & 3 \\
Triggering efficiency & & 3 \\
Total & 50 & 11 \\
\hline \hline
\end{tabular}


by about a factor of 4 . We additionally studied $d[A \Omega]_{\mathrm{eff}} /$ $d\left[\sigma_{\nu-N}\right]$ and find it to be approximately linear; for example, at $1 \mathrm{EeV}$, a $10 \%$ increase in $\sigma_{\nu-N}$ corresponded to a $10 \%$ increase in $[A \Omega]_{\mathrm{eff}}$.

For the Askaryan emission, AraSim implements a modified version of the model derived by Alvarez-Muñiz et al. [47]. A full description of modifications is provided elsewhere [31], but the primary differences arise due to the inclusion of the Landau-Pomeranchuk-Migdal effect by Alvarez-Muñiz but not by AraSim, and in AraSim's use of functional parametrizations for the shower profile instead of directly simulated shower profiles. The relative difference between waveform amplitudes produced by AraSim and those derived from a full shower Monte Carlo is at most $\sim 12 \%$ [48]. We conservatively estimate the effect of this systematic uncertainty by reducing or increasing the simulated field amplitude by $\pm 12 \%$ and assessing the change in $[A \Omega]_{\text {eff }}$ at the trigger level. The relative difference between the default parametrization and the scaled parametrization has a maximum value of about $25 \%$ near $10^{16} \mathrm{eV}$ and starts falling as energy increases. This is because at high energies the instrument acceptance becomes dominated by geometric effects (ray tracing, etc.) and not signal amplitude. At $10^{18} \mathrm{eV}$, the estimated uncertainties due to the Askaryan emission model are $-11 \% /+13 \%$.

In the second category of uncertainties, we consider those arising from our detector response and from measurements of quantities such as the index of refraction in ice and the attenuation length of radio waves in ice. These systematics are included in our calculation of the final limit. We consider uncertainties associated with (1) the attenuation length $\left(L_{\text {att }}\right)$ of South Pole Ice and (2) the depthdependent index of refraction $(n(z))$ of South Pole ice, (3) the calibration of the ARA signal chain, and (4) the triggering efficiency of the detector.

The model for the attenuation length $\left(L_{\text {att }}\right)$ of South Pole ice was derived from data taken with the ARA Testbed prototype [26]. Confidence bands providing an upper and lower limit on $L_{\text {att }}$ are given in the model. To set upper/ lower limits on our sensitivity, in AraSim, the upper and lower bounds for $L_{\text {att }}$ are substituted for the central value. At $10^{18} \mathrm{eV}$, the uncertainty on the analysis level effective area due to uncertainties in attenuation length are $-8 \% /+50 \%$.

The model for the depth-dependent index of refraction $n(z)$ was obtained by fitting data obtained by the RICE experiment [41]. The data was fitted with an exponential as a function of (negative) depth $z$ of the form $n_{d}-\left(n_{d}-n_{s}\right) e^{z \cdot n_{c}}$, finding the following parameter values and their respective uncertainties: $n_{d}=1.788 \pm 0.016$, $n_{s}=1.359 \pm 0.022$, and $n_{c}=0.0132 \pm 0.0017 \mathrm{~m}^{-1}$. We recalculate the sensitivity, setting all parameters to their upper and lower limits simultaneously. The lower (upper) limit generally corresponds to a slower (faster) transition from surface to deep ice and correspondingly has a smaller (larger) geometric acceptance for neutrinos. Additionally, since we do not change the ice-model assumption used to reconstruct the incoming direction of the rf emission as discussed in Sec. III D, this systematic uncertainty also captures errors which may be present if the true ice model for radio wave propagation does not match that used for reconstruction. At $10^{18} \mathrm{eV}$, the uncertainties on the analysis level effective area due to the index of refraction model are $5 \%$.

We consider four sources of uncertainties that exist in the signal chain. They are the transmission coefficient $t$ representing the impedance mismatch between the ice and the antenna, as well as between the antenna and the coaxial cable, the ambient noise power received $N_{\text {ant }}$, the signal chain noise power $N_{\mathrm{sc}}$, and the antenna directivity $D$. We follow the treatment used in the previous ARA result [32] where we consider the system signal-to-noise ratio representing the ratio of input signal power to total system noise power in a given channel,

$$
\mathrm{SNR}_{\mathrm{sys}}=\frac{t D P_{\mathrm{sig}}}{t N_{\mathrm{ant}}+N_{\mathrm{sc}}},
$$

with $P_{\text {sig }}$ being the received signal power. The four sources of uncertainty translate to an uncertainty in $\mathrm{SNR}_{\mathrm{sys}}$ by standard error propagation, which is then implemented as an uncertainty in the antenna gain $G$ in code $\left(\Delta G=\Delta \mathrm{SNR}_{\text {sys }} / P_{\text {sig }}\right)$. In line with previous ARA work, here we only consider the case where the effective gain of the instrument is reduced, providing a conservative estimate of our sensitivity. This is done because we lack sufficient calibration data at this time to constrain the upper bound on the gain. The VPol antenna gain has an overall estimated uncertainty of $-10 \%$, while the HPol antenna gain is estimated at $-32 \%$. The modified gain values are substituted in the simulation to assess the impact of this uncertainty, and the uncertainty at $10^{18} \mathrm{eV}$ is found to be $-3 \%$.

For the systematic uncertainty associated with the trigger efficiency of the detector as a function of RPR, $\epsilon$ (RPR), we compare the simulated trigger efficiency $\epsilon_{\text {sim }}(\mathrm{RPR})$ to the measured trigger efficiency in calibration pulser data $\epsilon_{\text {dat }}(\mathrm{RPR}): \Delta \epsilon=\epsilon_{\mathrm{dat}}(\mathrm{RPR})-\epsilon_{\mathrm{sim}}(\mathrm{RPR})$. We measure $\epsilon_{\text {dat }}(\mathrm{RPR})$ by varying a tunable attenuator on the local calibration pulsers described in Sec. II and counting the number of calibration pulsers recorded. Using AraSim, we find that the uncertainties on the trigger efficiency decreases the simulated $[A \Omega]_{\mathrm{eff}}$ from between $2 \%$ and $5 \%$ depending on energy, and at $10^{18} \mathrm{eV}$ the size of the effect is $-3 \%$.

We observed in previous calibration exercises that the stations trigger inefficiently on calibration pulsers whose direct ray-tracing solution intercepts the array at an angle steeper than $-25^{\circ}$ from horizontal; this can be seen in Ref. [49], where there is a deficiency of triggers in A2 and 
A3 after the pulser is lowered below $1300 \mathrm{~m}$ depth, despite the pulser being lowered to a total depth of $1700 \mathrm{~m}$. Therefore, for the calculation of $[A \Omega]_{\mathrm{eff}}$ used in the limit, we conservatively exclude neutrino simulated events with the same ray-tracing conditions. This results in a $~ 10 \%-$ $30 \%$ reduction in sensitivity, depending on energy. Excluding these steeply upgoing events is a conservative approach, as more exhaustive future studies might reveal that the cause of the trigger inefficiency to the calibration pulses does not have the same effect on neutrino events.

\section{DISCUSSION AND OUTLOOK}

In this paper, we present constraints on the flux of UHE neutrinos between $10^{16}$ and $10^{21} \mathrm{eV}$ from four years of data in A2 and A3. We have presented a description of the live time and the instrument, and detailed the cuts used to eliminate backgrounds in two complementary, blind analyses. The resultant limit from this search is the strongest limit set by ARA to date, and the strongest limit set by an in-ice radio neutrino detector above $10^{17} \mathrm{eV}$. The result utilizes more than quadruple the live time of the previously published ARA analysis and maintains reasonable efficiency to neutrinos while remaining general to signal shape and not requiring costly cuts on live time in Austral summer or angular cuts in the direction of anthropogenic sources like South Pole Station. We are encouraged that the two analyses, which leveraged complimentary sets of reconstruction and analysis tools, have similar sensitivity and produced consistent expected limits within $15 \%$ for all energy bins.

Post-unblinding, we were additionally able to further study our surface related backgrounds. As discussed previously, we observed zero events, consistent with our background estimates, including our estimated $10^{-3}$ events from above the surface. If we check the data taking runs that were excluded pre-unblinding because of the presence of large amounts of surface noise, we do observe a few events passing all cuts. We additionally are able to roughly estimate the probability of a single event of surface origin being misreconstructed as coming from within the ice. To do so, we take the product of the fraction of runs in which we observe only one surface event and multiply by our estimated misreconstruction rate. We estimate the misreconstruction rate by taking the ratio of the number of events reconstructing inside the ice, relative to those reconstructing outside the ice, in the surface noisy runs. For example, in A3, we find that there may be approximately 0.2 such "misreconstructing singlets." We note that this estimate is biased to larger values, because in order to measure the misreconstruction rate, we rely on the number of events reconstructing inside the ice in runs which demonstrate large amounts of surface noise, and were decided preunblinding to be unfit for analysis. These two postunblinding studies demonstrate the role of the surfacenoisy cut in the present analysis and represent an opportunity for growth during the development of future reconstruction techniques.

We underscore several important features of this newest result. First, it demonstrates ARA's capability to analyze its growing data set. Compared to our previous result, which analyzed the first ten months of data from stations A2 and A3 [32], this analysis leverages data from four years of data taking in each of the two stations. After removing intermittent periods of downtime, we have about 1100 days (75\%) of live time that was good for analysis for each station. This amounts to 2162 days of combined live time. This analysis is therefore the first ARA result to analyze $\mathcal{O}(10)$ station years of data. This demonstrates the capability to analyze our growing data set, which will be important as ARA looks to the future. There is roughly 4080 additional days of live time awaiting analysis on archive, with the analysis pending ongoing calibration efforts. With the full five-station ARA array collecting data since January 2018, the data set is expected to roughly double again by 2022 (total of approximately $11 \mathrm{k}$ days of live time). In Fig. 7, we additionally show the projected triggerlevel single-event sensitivity that the five-station ARA5 array can achieve with data that will have been accumulated through 2022. As can be seen, ARA is poised to be the leading UHE neutrino detector above $10^{17} \mathrm{eV}$; the IceCube and Auger experiments will also accumulate additional live time amounting to about $40 \%$ and $25 \%$ increases over their respective published limits.

Second, the analysis maintains reasonable efficiency $\left(\sim 35 \%\right.$ at $10^{18} \mathrm{eV}$, and reaching $50 \%$ efficiency near a voltage SNR of 6) while remaining general and not relying on quantities that are strongly model dependent, such as a correlation with a signal template. This is advantageous because although the Askaryan signal has been observed in the laboratory and in the atmosphere from cosmic-ray air showers [51-53], it has never before been observed in a dense media in nature.

In line with our previous two-station result [32], this analysis did not require excluding data recorded during the Austral summer, nor did it require geometric rejection regions specifically in the direction of the South Pole. In the prior analyses of the prototype station [31,38], 31\% of live time was lost due to anthropogenic activities during the Austral summer, as well as $9 \%$ due to the detector's solid angle coverage in directions near the South Pole.

We note three challenges overcome in these analyses that have resulted in improvements moving forward, especially as the ARA data set continues to grow, the diversity of the array increases, and the field looks forward to a large-scale radio array in IceCube-Gen2. The first challenge was managing the time-dependent nature of the ARA instruments. Some of the time-dependent nature is owed to the different data taking configurations, as described in the Appendix, and often reflect improved understanding of the instrument and the ice. For example, an early trigger 
configuration led to triggering signals being off-center in the digitized waveform, and this was later corrected. The change to the readout length was made after Monte Carlo studies revealed that longer readout windows increased the probability with which a station records both the direct and refracted/reflected pulse that are possible because of the depth-dependent index of refraction. Since learning from these processes, we have reached more stable operations configurations and are working on additional streamlining. Some time dependence is owed to changing detector characteristics; for example, for some periods of time in ARA station 3, a digitization board exhibited a high amount of readout noise. Such time-dependent detector features required adjustments to analysis algorithms and analysis thresholds. As a result of the analyses described herein, identification of such time periods has also been considerably streamlined.

The second challenge was improvement in intra-collaboration communication between the ARA operations and analysis teams. In many cases, periods of live time that were contaminated with calibration activity were recorded in operations reports, but were only later accounted for the analyses. We plan to work to streamline this pipeline for future ARA analyses.

The third challenge was managing anthropogenic activity from the South Pole over several Austral summers. Despite most human activity being isolated nearly two miles away, the analysis requires aggressive cuts on downgoing signals, which eliminated $10 \%-30 \%$ of neutrino events. Improvements to reconstruction algorithms to more confidently reject downgoing events without requiring such substantial cuts on solid angle, or to more confidently reconstruct events with low hit multiplicity, will improve the analysis efficiencies in the future.

\section{ACKNOWLEDGMENTS}

The main authors of this paper were Brian Clark, MingYuan Lu, and Jorge Torres, with Brian Clark and MingYuan Lu leading the data analysis for this result. The ARA Collaboration designed, constructed, and now operates the ARA detectors. Data processing and calibration, Monte Carlo simulations of the detector and of theoretical models and data analyses were performed by a large number of collaboration members, who also discussed and approved the scientific results presented here. We are grateful for contributions and discussions from Lucas Smith and Suren Gourapura. We are thankful to the National Science Foundation (NSF) Office of Polar Programs and Physics Division for funding support through Grants No. 1806923, No. 1404266, No. OPP-902483, No. OPP-1359535, and No. 1607555. We further thank the Taiwan National Science Councils Vanguard Program NSC 92-2628-M-002-09 and the Belgian F. R. S.-FNRS Grant No. 4.4508.01. We also thank the University of Wisconsin Alumni Research Foundation, the University of
Maryland, and the Ohio State University for their support. B. A. C. thanks the NSF for support through the Graduate Research Fellowship Program Grant No. DGE-1343012 and the Astronomy and Astrophysics Postdoctoral Fellowship under Grant No. 1903885, as well as the Institute for Cyber-Enabled Research at Michigan State University. A. C. thanks the NSF for CAREER Grants No. 1255557 and No. GRT00049285 and also the Ohio Supercomputer Center. K. D. H. likewise thanks the NSF for their support through CAREER Grant No. 0847658. S. A. W. thanks the NSF for support through CAREER Grant No. 1752922 and the Bill and Linda Frost Fund at the California Polytechnic State University. A. C., H. L., and D. Z. B. thank the United States-Israel Binational Science Foundation for their support through Grant No. 2012077. A.C., A. K., and J.L.K. thank the NSF for the support through BIGDATA Grant No. 1250720. D. Z. B. and A. N. acknowledge support from National Research Nuclear University MEPhi (Moscow Engineering Physics Institute). K. H. thanks the NSF for support through the Graduate Research Fellowship Program Grant No. DGE1746045. A. G. V. thanks the Sloan Foundation and the Research Corporation for Science Advancement. R. J. N. thanks the Leverhulme Trust for their support. K. D. de V. is supported by European Research Council under the European Union's Horizon 2020 research and innovation program (Grant No. 805486). Finally, we are thankful to the Raytheon Polar Services Corporation, Lockheed Martin, and the Antarctic Support Contractor for field support and enabling our work on the harshest continent.

\section{APPENDIX: LIMIT CALCULATION}

We set a 90\% confidence level upper limit on the flux $E_{i} F(E)_{i}$ in the $i$ th energy bin of width $\operatorname{dlog}_{10} E$ according to Eq. (A1),

$$
E_{i} F(E)_{i}=\frac{n_{i}}{\Lambda_{i} \ell n(10) \operatorname{dlog}_{10} E},
$$

where $n_{i}$ is the Feldman-Cousins upper limit for zero measured events on a background of zero, accounting for the systematic uncertainties (added in quadrature) described in Sec. V. This is done according to the prescription in Conrad et al. [54] with the improvements suggested by Hill [55]. Note that in the absence of uncertainties, $n_{i}=2.44$, as commonly observed in the literature [56]. Use of zero instead of the actual background estimate for the analysis setting the limit is conservative by $\sim 2 \%$ and does not substantially change the result.

We take d $\log _{10} E=1$, corresponding to decade wide bins in energy. $\Lambda_{i}$ is the exposure of the instrument summed over stations and configurations, taking into account analysis efficiencies as presented in Fig. 8. $\Lambda_{i}$ for a given energy bin is defined explicitly as 
TABLE II. Configuration definitions for A2 and A3, highlighting their various trigger parameters and live times.

\begin{tabular}{|c|c|c|c|c|c|c|}
\hline Station & Config & Readout window (ns) & Trigger window (ns) & Active delays & Live time (days) & Num events (million) \\
\hline \multirow{5}{*}{2} & 1 & 400 & 110 & Yes & 179 & 108.9 \\
\hline & 2 & 400 & 110 & No & 142 & 97.3 \\
\hline & 3 & 400 & 110 & Yes & 94 & 54.5 \\
\hline & 4 & 520 & 170 & Yes & 439 & 216.8 \\
\hline & 5 & 520 & 170 & No & 287 & 129.7 \\
\hline \multirow{5}{*}{3} & 1 & 400 & 110 & Yes & 79 & 43.7 \\
\hline & 2 & 400 & 110 & No & 147 & 114.2 \\
\hline & 3 & 520 & 170 & Yes & 345 & 207.0 \\
\hline & 4 & 520 & 170 & No & 260 & 171.8 \\
\hline & 5 & 400 & 110 & Yes & 191 & 118.8 \\
\hline
\end{tabular}

$$
\Lambda_{i}=\sum_{j_{\text {stations }}=1}^{2} \sum_{k_{\mathrm{configs}}=1}^{5} \epsilon_{i, j, k}[A \Omega]_{\mathrm{eff}, i, j, k} T_{j, k}
$$

where for a specific energy $(i)$, station $(j)$, and configuration $(k), \epsilon_{i, j, k}$ is the efficiency and $[A \Omega]_{\mathrm{eff}, i, j, k}$ is the effective area as shown in Fig. 5. $T_{j, k}$ is the live time of the instrument for a specific station and configuration as reported in Table II.

The effective areas $[A \Omega]_{\mathrm{eff}}$ of the instruments are computed from the effective volumes $[V \Omega]_{\mathrm{eff}}$ through the thin-target approximation,

$$
[A \Omega]_{\mathrm{eff}}=\frac{[V \Omega]_{\mathrm{eff}}}{\mathcal{L}_{\mathrm{int}}},
$$

where $\mathcal{L}_{\text {int }}=m_{N} /\left(\rho \sigma_{\nu-N}\right)$ is the interaction length of a neutrino in the Earth,

where $m_{N}=1.67 \times 10^{-24} \mathrm{~g}$ is the mass of a nucleon, $\rho=0.92 \mathrm{~g} / \mathrm{cm}^{3}$ is the average density of ice, and $\sigma_{\nu-N}$ is the neutrino/antineutrino-nucleon cross section in the units of $\mathrm{cm}^{2}$ as computed in Connolly et al. [18]. The effective volumes are calculated by Monte Carlo methods. Using AraSim at specific energy, we simulate a number of neutrinos $N_{\text {thrown }}$ in an interaction volume $V_{\text {thrown }}$ with an isotropic distribution of arrival directions, and with an equal number of events between the three neutrino flavors and between neutrinos/antineutrinos. The sum of the weights of triggered events, $\sum w_{\text {trig }}$, determines the effective volume,

$$
[V \Omega]_{\mathrm{eff}}=\frac{\sum w_{\text {trig }}}{N_{\text {thrown }}} \times V_{\text {thrown }} \times 4 \pi,
$$

where the weighting accounts for the neutrino survival probability up to the interaction vertex.

\section{Limit with alternative flux scaling}

In Fig. 10, we show the same 90\% CL limit as we presented in Fig. 7, but with an alternative scaling on the y axis so that the flux is multiplied by an additional factor of energy in $\mathrm{GeV}$. In this scaling, the y axis represents energy flux per $\mathrm{cm}^{2} \mathrm{~s}^{-1} \mathrm{sr}^{-1}$ as opposed to the particle flux.

\section{Live time configurations}

In Table II, we provide a table of the different station configurations, outlining the key parameters that differentiate them, along with the quantity of live time for which they were active and the total number of events recorded (in millions). Note that A3 configuration 5 varies from A3 configuration 1 by the loss of quality digitizer data in string 4. The trigger window is the amount of time during which 3/8 same-polarization antennas must have coincident single-channel triggers to trigger the readout of the instrument. The readout window is the length of time for which the digitizers are read out. The active delays column represents whether a set of trigger delays were applied to account for different cable lengths from different channels or not.

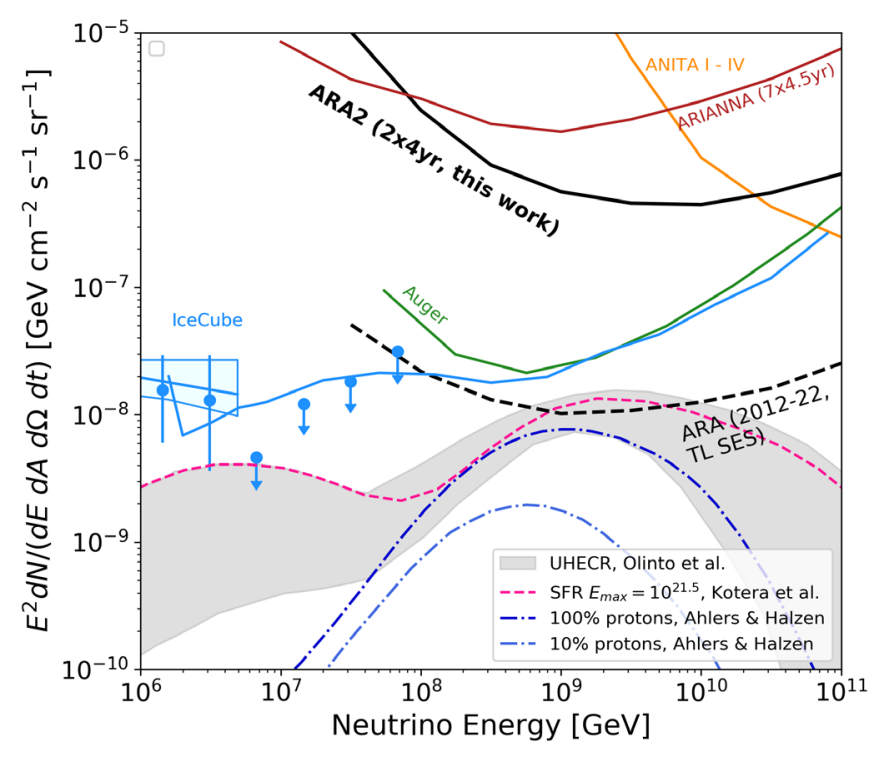

FIG. 10. Same as Fig. 7, but with an alternative flux scaling. 

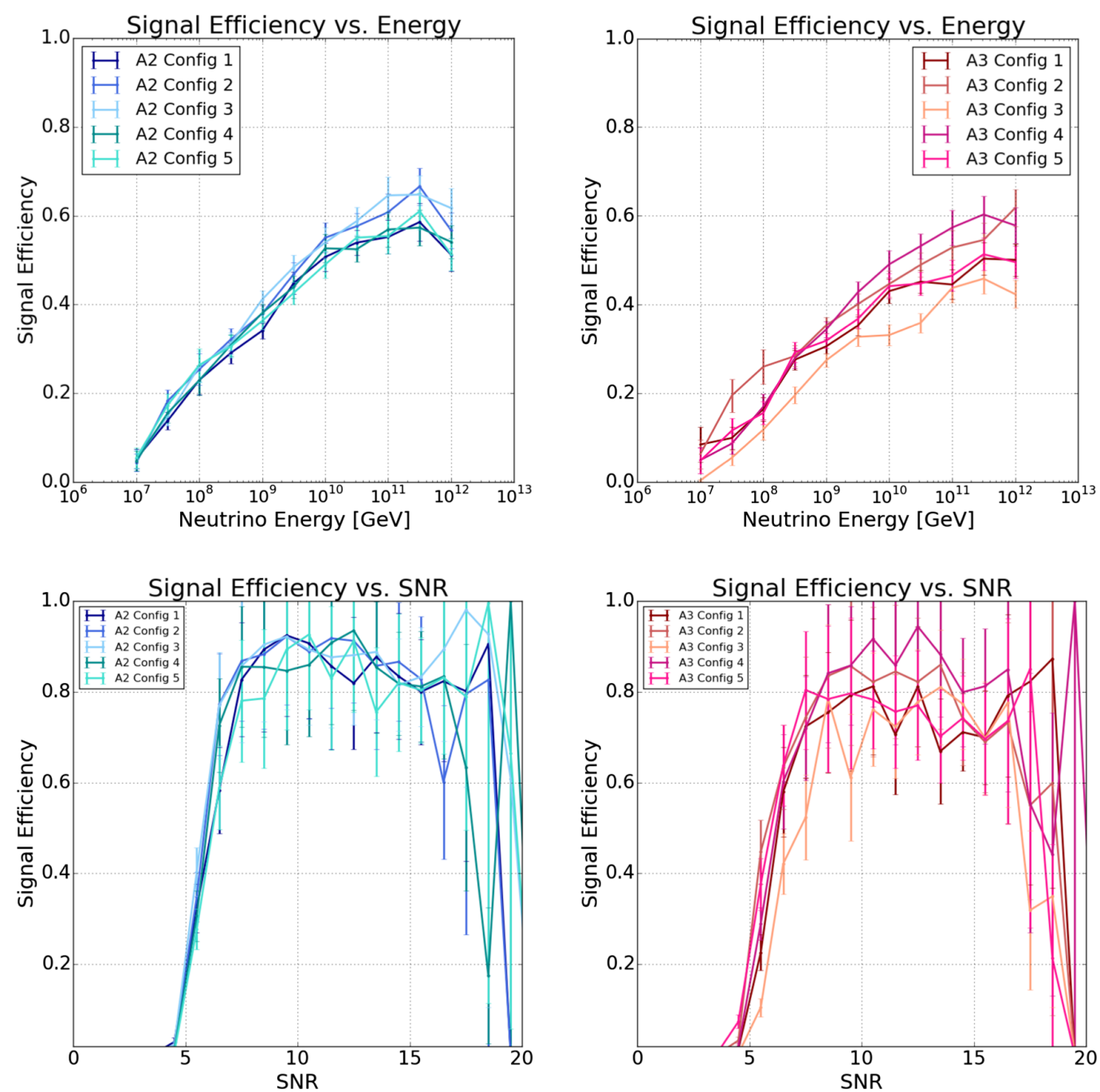

FIG. 11. Signal efficiency from Analysis A for each configuration in A2 and A3, respectively. Top row: signal efficiency as a function of neutrino energy for A2 (top left) and A3 (top right). Bottom row: signal efficiency as a function of signal-to-noise-ratio for A2 (bottom left) and A3 (bottom right) assuming the same energy weighting scheme as in Fig. 8.

In Fig. 11, we present the analysis efficiency from Analysis A for each configuration in both stations, as estimated using AraSim simulation. The analysis efficiency is driven by the analysis cuts, which depend on the background distribution observed during the live time of each configuration.
[1] K. Greisen, End to the Cosmic-Ray Spectrum? Phys. Rev. Lett. 16, 748 (1966).

[2] G. T. Zatsepin and V.A. Kuzmin, Upper limit of the spectrum of cosmic rays, Pis'ma Zh. Eksp. Teor. Fiz. 4, 114 (1966) [JETP Lett. 4, 78 (1966)], http://www.jetpletters .ac.ru/ps/1624/article_24846.shtml.

[3] B. L. Berman and S.C. Fultz, Measurements of the giant dipole resonance with monoenergetic photons, Rev. Mod. Phys. 47, 713 (1975).
[4] R. J. Gould and G. P. Schréder, Opacity of the Universe to high-energy photons, Phys. Rev. 155, 1408 (1967).

[5] E. Waxman and J. N. Bahcall, Neutrino afterglow from gamma-ray bursts: $\sim 1018 \mathrm{eV}$, Astrophys. J. 541, 707 (2000).

[6] K. Murase, Active galactic nuclei as high-energy neutrino sources, in Neutrino Astronomy: Current Status, Future Prospects, edited by T. Gaisser and A. Karle (World Scientific, Singapore, 2017), pp. 15-31. 
[7] M. G. Aartsen et al. (IceCube Collaboration), A combined maximum-likelihood analysis of the high-energy astrophysical neutrino flux measured with IceCube, Astrophys. J. 809, 98 (2015).

[8] M. G. Aartsen et al. (Liverpool Telescope, MAGIC, H.E.S.S., AGILE, Kiso, VLA/17B-403, INTEGRAL, Kapteyn, Subaru, HAWC, Fermi-LAT, ASAS-SN, VERITAS, Kanata, IceCube, and Swift NuSTAR Collaborations), Multimessenger observations of a flaring blazar coincident with high-energy neutrino IceCube-170922A, Science 361, eaat1378 (2018).

[9] M. G. Aartsen et al. (IceCube Collaboration), Neutrino emission from the direction of the blazar TXS 0506+056 prior to the IceCube-170922A alert, Science 361, 147 (2018).

[10] V. S. Berezinsky and G. T. Zatsepin, Cosmic rays at ultra high energies (neutrino?), Phys. Lett. 28B, 423 (1969).

[11] D. Hooper, A. Taylor, and S. Sarkar, The impact of heavy nuclei on the cosmogenic neutrino flux, Astropart. Phys. 23, 11 (2005).

[12] D. Allard, M. Ave, N. Busca, M. A. Malkan, A. V. Olinto, E. Parizot, F. W. Stecker, and T. Yamamoto, Cosmogenic neutrinos from the propagation of ultrahigh energy nuclei, J. Cosmol. Astropart. Phys. 09 (2006) 005.

[13] K. Kotera, D. Allard, and A. V. Olinto, Cosmogenic neutrinos: parameter space and detectabilty from $\mathrm{PeV}$ to ZeV, J. Cosmol. Astropart. Phys. 10 (2010) 013.

[14] K. Kotera and A. V. Olinto, The astrophysics of ultrahighenergy cosmic rays, Annu. Rev. Astron. Astrophys. 49, 119 (2011).

[15] A. van Vliet, J. R. Hörandel, and R. Alves Batista, Cosmogenic gamma-rays and neutrinos constrain UHECR source models, Proc. Sci., ICRC2017 (2018) 562.

[16] M. Ahlers and F. Halzen, Minimal cosmogenic neutrinos, Phys. Rev. D 86, 083010 (2012).

[17] J. K. Thomas, R. Moharana, and S. Razzaque, Ultrahigh energy neutrino afterglows of nearby long duration gammaray bursts, Phys. Rev. D 96, 103004 (2017).

[18] A. Connolly, R. S. Thorne, and D. Waters, Calculation of high energy neutrino-nucleon cross sections and uncertainties using the Martin-Stirling-Thorne-Watt parton distribution functions and implications for future experiments, Phys. Rev. D 83, 113009 (2011).

[19] A. Cooper-Sarkar, P. Mertsch, and S. Sarkar, The high energy neutrino cross-section in the Standard Model and its uncertainty, J. High Energy Phys. 08 (2011) 042.

[20] M. G. Aartsen et al. (IceCube Collaboration), Differential limit on the extremely-high-energy cosmic neutrino flux in the presence of astrophysical background from nine years of IceCube data, Phys. Rev. D 98, 062003 (2018).

[21] A. Aab et al. (Pierre Auger Collaboration), Probing the origin of ultra-high-energy cosmic rays with neutrinos in the EeV energy range using the Pierre Auger Observatory, J. Cosmol. Astropart. Phys. 10 (2019) 022.

[22] S. Buitink, O. Scholten, J. Bacelar, R. Braun, A. G. de Bruyn, H. Falcke, K. Singh, B. Stappers, R. G. Strom, and R. al Yahyaoui, Constraints on the flux of ultra-high energy neutrinos from Westerbork Synthesis Radio Telescope observations, Astron. Astrophys. 521, A47 (2010).
[23] P. W. Gorham et al. (ANITA Collaboration), Constraints on the ultrahigh-energy cosmic neutrino flux from the fourth flight of ANITA, Phys. Rev. D 99, 122001 (2019).

[24] A. Anker et al., A search for cosmogenic neutrinos with the ARIANNA test bed using 4.5 years of data, J. Cosmol. Astropart. Phys. 03 (2020) 053.

[25] J. Álvarez-Muñiz et al. (GRAND Collaboration), The giant radio array for neutrino detection (GRAND): Science and design, Sci. China Phys. Mech. Astron. 63, 219501 (2020).

[26] P. Allison et al., Design and initial performance of the Askaryan Radio Array prototype EeV neutrino detector at the South Pole, Astropart. Phys. 35, 457 (2012).

[27] G. A. Askar'yan, Excess negative charge of an electronphoton shower and its coherent radio emission, Zh. Eksp. Teor. Fiz. 41, 616 (1961) [Sov. Phys. JETP 14, 441 (1962)], http://www.jetp.ac.ru/cgi-bin/e/index/e/14/2/p441?a=list.

[28] G. A. Askar'yan, Coherent radio emission from cosmic showers in air and in dense media, Sov. J. Exp. Theor. Phys. 21, 658 (1965), http://www.jetp.ac.ru/cgi-bin/e/index/e/21/ 3/p658?a=list.

[29] P. W. Gorham et al. (ANITA Collaboration), Observations of the Askaryan Effect in Ice, Phys. Rev. Lett. 99, 171101 (2007).

[30] S. Barwick, D. Besson, P. Gorham, and D. Saltzberg, South Polar in situ radio-frequency ice attenuation, J. Glaciol. 51, 231 (2005).

[31] P. Allison et al. (ARA Collaboration), First constraints on the ultra-high energy neutrino flux from a prototype station of the Askaryan Radio Array, Astropart. Phys. 70, 62 (2015).

[32] P. Allison et al. (ARA Collaboration), Performance of two Askaryan Radio Array stations and first results in the search for ultrahigh energy neutrinos, Phys. Rev. D 93, 082003 (2016).

[33] Specifications for the IceRay Sampler (IRS) ASI, https:// www.phys.hawaii.edu/ varner/IRS_spec_v01.pdf (2009).

[34] G. S. Varner, L. L. Ruckman, P. W. Gorham, J. W. Nam, R. J. Nichol, J. Cao, and M. Wilcox, The large analog bandwidth recorder and digitizer with ordered readout (LABRADOR) ASIC, Nucl. Instrum. Methods Phys. Res., Sect. A 583, 447 (2007).

[35] J. M. Roberts, E. Oberla, P. Allison, G. S. Varner, S. Spack, B. Fox, and B. Rotter, LAB4D: A low power, multi-GSa/s, transient digitizer with sampling timebase trimming capabilities, Nucl. Instrum. Methods Phys. Res., Sect. A 925, 92 (2019).

[36] M. G. Aartsen et al. (IceCube Collaboration), Probing the origin of cosmic rays with extremely high energy neutrinos using the IceCube Observatory, Phys. Rev. D 88, 112008 (2013).

[37] M.-Y. Lu, C. G. Pfendner, and A. Shultz (ARA Collaboration), Ultra-high energy neutrino search with the Askaryan Radio Array, Proc. Sci., ICRC2017 (2018) 966.

[38] P. Allison et al. (ARA Collaboration), Constraints on the ultra-high-energy neutrino flux from Gamma-Ray bursts from a prototype station of the Askaryan radio array, Astropart. Phys. 88, 7 (2017).

[39] P. Allison et al. (ARA Collaboration), Observation of reconstructable radio emission coincident with an $\mathrm{x}$-class solar flare in the Askaryan radio array prototype station, arXiv:1807.03335. 
[40] A. Romero-Wolf et al., An interferometric analysis method for radio impulses from ultra-high energy particle showers, Astropart. Phys. 60, 72 (2015).

[41] I. Kravchenko, D. Besson, and J. Meyers, In situ index-ofrefraction measurements of the South Polar firn with the RICE detector, J. Glaciol. 50, 522 (2004).

[42] P. Schellart et al., Detecting cosmic rays with the LOFAR radio telescope, Astron. Astrophys. 560, A98 (2013).

[43] B. Dailey, Analysis of the second flight of the antarctic impulsive transient antenna with a focus on filtering techniques, Ph.D. Thesis, The Ohio State University, 2017.

[44] P. W. Gorham et al. (ANITA Collaboration), Constraints on the diffuse high-energy neutrino flux from the third flight of ANITA, Phys. Rev. D 98, 022001 (2018).

[45] M. G. Aartsen et al. (IceCube Collaboration), Observation and characterization of a cosmic muon neutrino flux from the northern hemisphere using six years of IceCube data, Astrophys. J. 833, 3 (2016).

[46] P. Allison et al., Design and performance of an interferometric trigger array for radio detection of high-energy neutrinos, Nucl. Instrum. Methods Phys. Res., Sect. A 930, 112 (2019).

[47] J. Alvarez-Muniz, A. Romero-Wolf, and E. Zas, Practical and accurate calculations of Askaryan radiation, Phys. Rev. D 84, 103003 (2011).

[48] E. Hong, Searching for ultra-high energy neutrinos with data from a prototype station of the askaryan radio array, Ph.D. Thesis, The Ohio State University, 2014.
[49] P. Allison et al. (ARA Collaboration), Long-baseline horizontal radio-frequency transmission through polar ice, arXiv:1908.10689.

[50] A. V. Olinto, K. Kotera, and D. Allard, Ultrahigh energy cosmic rays and neutrinos, Nucl. Phys. B, Proc. Suppl. 217, 231 (2011).

[51] A. Bellćtoile, R. Dallier, A. Lecacheux, V. Marin, L. Martin, B. Revenu, and D. Torres, Evidence for the charge-excess contribution in air shower radio emission observed by the CODALEMA experiment, Astropart. Phys. 69, 50 (2015).

[52] A. Aab et al. (Pierre Auger Collaboration), Probing the radio emission from air showers with polarization measurements, Phys. Rev. D 89, 052002 (2014).

[53] O. Scholten et al., Measurement of the circular polarization in radio emission from extensive air showers confirms emission mechanisms, Phys. Rev. D 94, 103010 (2016).

[54] J. Conrad, O. Botner, A. Hallgren, and C. Perez de los Heros, Including systematic uncertainties in confidence interval construction for Poisson statistics, Phys. Rev. D 67, 012002 (2003).

[55] G. C. Hill, Comment on "Including systematic uncertainties in confidence interval construction for Poisson statistics", Phys. Rev. D 67, 118101 (2003).

[56] G. J. Feldman and R. D. Cousins, Unified approach to the classical statistical analysis of small signals, Phys. Rev. D 57, 3873 (1998). 